\title{
Skeletal geometry and niche transitions restore organ size and shape during zebrafish fin regeneration
}

10

Scott Stewart ${ }^{1, *, \#}$, Gabriel A. Yette ${ }^{1,2, \#}$, Heather K. Le Bleu ${ }^{1,2}$, Astra L. Henner ${ }^{1}$, Joshua A. Braunstein $^{1,2}$, Jad W. Chehab ${ }^{1}$, Michael J. Harms ${ }^{1,3}$ and Kryn Stankunas ${ }^{1,2, *}$

15

Short title: Skeletal geometry as positional information for fin regeneration

$25{ }^{1}$ Institute of Molecular Biology

${ }^{2}$ Department of Biology

${ }^{3}$ Department of Chemistry and Biochemistry

University of Oregon

273 Onyx Bridge

301318 Franklin Blvd

Eugene, OR 97403-1229

Office: (541) 346-7416

Fax: (541) 346-4854

35

* Correspondence: kryn@uoregon.edu; sstewar2@uoregon.edu

\# Equal contributions

40 


\section{ABSTRACT}

Regenerating fish fins return to their original size and shape regardless of the nature or extent of injury. Prevailing models for this longstanding mystery of appendage regeneration speculate fin

45 cells maintain uncharacterized positional identities that instruct outgrowth after injury. Using zebrafish, we find differential Wnt production correlates with the extent of regeneration across the caudal fin. We identify Dachshund transcription factors as markers of distal blastema cells that produce Wnt and thereby promote a pro-progenitor and -proliferation environment. We show these Dach-expressing "niche cells" derive from mesenchyme populating cylindrical and

50 progressively tapered fin rays. The niche pool, and consequently Wnt, steadily dissipates as regeneration proceeds; once exhausted, ray and fin growth stops. Supported by mathematical modeling, we show longfin ${ }^{t 2}$ zebrafish regenerate exceptionally long fins due to a perdurant niche, representing a "broken countdown timer". We propose regenerated fin size is dictated by the amount of niche formed upon damage, which simply depends on the availability of intra-ray

55 mesenchyme defined by skeletal girth at the injury site. Likewise, the fin reestablishes a tapered ray skeleton because progenitor osteoblast output reflects diminishing niche size. This "transpositional scaling" model contends mesenchyme-niche state transitions and positional information provided by self-restoring skeletal geometry rather than cell memories determine a regenerated fin's size and shape. 


\section{MAIN TEXT}

Regenerating organs restore their original size and shape after injury. Vertebrate appendage regeneration, including that of teleost fish fins, provides a striking example of this phenomenon. Major fin amputations, tiny resections, and cuts of diverse geometry all produce

65 the same outcome - a restored fin matching the original's form and in scale with the animal's body. Spallanzani, Broussonet, and T. H. Morgan pioneered studies of this longstanding mystery of regeneration in the $18^{\text {th }}$ and $19^{\text {th }}$ centuries (Broussonet, 1786; Morgan, 1900). For example, Morgan used oblique caudal fin resections to show that regeneration rates initially correlate with the amount of tissue lost and then progressively slow, ultimately stopping growth when the

70 original size is regained (Morgan, 1900).

Prevailing models, now largely from zebrafish studies, posit that fin tissue adjacent to damage sites interpret Cartesian coordinate-like positional information to trigger the appropriate rate and extent of re-growth (Nachtrab et al., 2013; Rabinowitz et al., 2017; Rolland-Lagan et al., 2012; Tornini et al., 2016; Wolpert, 2016). This concept supposes an extensive array of

75 positional identities, some epigenetic mechanism for cells to store such identities, and, perhaps most puzzling, for the positional information to be restored during the regeneration process. Ultimately, however, the rate and extent of outgrowth is dictated by production of growth factors, including FGF (Lee et al., 2005; Poss et al., 2000; Shibata et al., 2016) and Wnt (Stewart et al., 2014; Stoick-Cooper et al., 2007; Wehner et al., 2014), with the net effect of promoting

80 cellular proliferation. Therefore, understanding growth factor production dynamics provides a logical entry to uncover fin size and shape restoration mechanisms.

Progressive fin regenerative outgrowth depends on spatially segregated growth factor production within an organized blastema. The blastema comprises heterogeneous cell types and 
forms soon after amputation by de-differentiation of mature cells and their migration into a

85 cavity defined by the enveloping wound epidermis (Wehner and Weidinger, 2015). The blastema then arranges by both cell type and cell state, with distal progenitor and proximal differentiating zones. De-differentiated progenitor osteoblasts (pObs) migrate to blastema peripheries and hierarchically arrange with most progenitor state cells distally enriched (Stewart et al., 2014). Differentiating osteoblasts derived from the pOb pool locate more proximally and continue

90 maturing to progressively extend re-forming bony rays. The central core of the blastema is largely comprised of "activated" mesenchymal cells that spill out from injury-exposed intra-ray space (Tornini et al., 2016; Tornini et al., 2017). These mesenchymal cells are the likely source of a distal, specialized "orchestrating center" (Wehner et al., 2014) that generates a pro-growth environment, including by producing mitogenic FGF (Lee et al., 2005; Poss et al., 2000; Shibata

95 et al., 2016) and pro-progenitor Wnt (Lee et al., 2009; Stewart et al., 2014; Stoick-Cooper et al., 2007; Wehner et al., 2014). An even further distal, small pool of distinct blastema cells may serve as a largely quiescent reservoir (Nechiporuk and Keating, 2002). Distal blastema progenitor-supporting activity is balanced by proximal pro-differentiation signals, including BMP and Wnt-opposing Dkk1 produced by pObs that transitioned from a progenitor to 100 differentiating state (Stewart et al., 2014).

Wnt signals indirectly promote cell proliferation (Wehner et al., 2014), in part by maintaining pObs (Stewart et al., 2014), to drive outgrowth until the fin is fully restored. Wnts such as $w n t 5 a, w n t 5 b$, and wnt10a are produced in the regenerating fin (Lee et al., 2009; Stewart et al., 2014; Stoick-Cooper et al., 2007; Wehner et al., 2014) including by distal blastema cells.

105 We hypothesized differential Wnt production determines the rate and ultimately extent of regeneration. Revisiting Morgan's experiments (Morgan, 1900), we obliquely amputated adult 
zebrafish fins from the distal tip of the dorsal side to a proximal location ventrally (see Fig. S1 for fin amputation planes and anatomical definitions). We then variably inhibited Wnt secretion by graded dosing of low concentrations of Wnt-C59 (Proffitt et al., 2013; Stewart et al., 2014).

110 Partial perturbation of Wnt signaling prevented dorsal but not ventral tissue regeneration (Fig. 1A-E). Similarly, central tissue was more sensitive than peripheral tissue to Wnt inhibition following perpendicular resections (Fig. S2). We infer differential Wnt production underlies reacquisition of the stereotypical shape of a zebrafish fin. Concordantly, levels of wnt5a, a representative distal blastema-expressed Wnt (Lee et al., 2009; Stewart et al., 2014; Stoick-

115 Cooper et al., 2007; Wehner et al., 2014), correlated with the demand for regenerative growth along the proximal-distal axis four days after oblique fin amputations (Fig. 1F, G).

Wnt signaling "strength" could be encoded either by the amount of Wnt produced per distal blastema cell or the number of Wnt-producing cells. Regardless, we reasoned that understanding the properties of this unique population was key to deciphering size control mechanisms. Hereafter, we term these "niche cells" for brevity and because they generate the pro-progenitor and pro-growth environment that enables tissue expansion (Morrison and Spradling, 2008). We used RNA-Seq of 4 days post amputation (dpa) distal vs. proximal fin regenerates, as demarcated by the distal epidermal marker $\operatorname{Tg}($ shha:EGFP) (Ertzer et al., 2007), to identify niche-characterizing factors (Fig. S3). Among transcription factors, the dachshund 125 (dach) family members (Mardon et al., 1994; Shen and Mardon, 1997) dacha (5.9-fold) and dachc (6.0-fold) were notably distally enriched (Fig. S3). We also used the RNA-Seq dataset to confirm the distal enrichment of several Wnts, with particularly high levels of wnt5a and wnt5b (Fig. S3). dachc mimicked $w n t 5 a$ in having notably higher expression levels in 4 dpa proximal tissue of diagonally amputated regenerating fins (Fig. 1H, I). 

(hereafter referred to as Dach) over the course of fin regeneration (Fig. 1J-O). Dach-expressing cells were rarely observed before 3 dpa (Fig. 1J). At 3 dpa, Dach became robustly expressed in distal cells adjacent to, but distinct from, Runx $2^{+}$pre-osteoblasts (Fig. 1K). Dach $^{+}$niche cells then steadily reduced over the course of regeneration to a small residual population also found in 135 distal tissue of unamputated fins (Fig. 1L-O). We conclude Dach transcription factors mark Wntproducing niche cells throughout the course of regeneration. The three-day delay prior to the appearance of $\mathrm{Dach}^{+}$niche corresponds with the view that fin regeneration proceeds by an acute injury repair phase followed by a prolonged period of progressive outgrowth (Wehner and Weidinger, 2015). Finally, differential numbers of niche cells produced upon injury and then 140 maintained through regeneration likely accounts for our observation that variable Wnt strength correlates with extent of fin outgrowth.

Wnt/ $\beta$-catenin signaling is active in niche cells where it indirectly promotes further proximal mesenchyme proliferation (Wehner et al., 2014). However, a potential role for Wnt in maintaining the niche pool itself is unexplored. Using the new Dach marker, we found that pan-

145 Wnt inhibition initiated at 4 dpa using Wnt-C59 depleted niche cells (Fig. S4). Wnt inhibition prevented outgrowth but did not disrupt osteoblast differentiation, joint formation, or skeletal maturation (Fig. S4), reinforcing our previous insight that $\mathrm{Wnt} / \beta$-catenin promotes bone progenitor maintenance upstream of and in opposition to differentiation (Stewart et al., 2014). Wnt, whether canonical or non-canonical, appears to have an analogous autocrine role 150 maintaining the niche population in a state whereby it can drive continued regeneration.

The loss of niche cells upon Wnt inhibition allowed us to test if artificial depletion of the niche pool mimics normal termination of regeneration - as supported by our observation that 
$\mathrm{Dach}^{+}$niche cells progressively decrease over regeneration. Wnt inhibition irreversibly blocked fin regeneration, with only rare outgrowth seen long after drug removal (Fig. S4). In contrast, re155 amputating Wnt-inhibited fins re-initiated full regeneration. Small molecule inhibition of FGF receptor signaling prevented outgrowth to the same degree as Wnt inhibition (Fig. S4). However, as FGF inhibition did not deplete $\mathrm{Dach}^{+}$niche cells (Fig. S4), regeneration resumed following drug washout, matching previous observations (Lee et al., 2005). Therefore, mitogenic FGF, which appears niche-expressed (Lee et al., 2005; Poss et al., 2000; Shibata et al., 2016) and is 160 Wnt-dependent (Wehner et al., 2014), acts downstream of Wnt's niche maintenance role. We propose niche cell numbers set dynamic levels of both signaling proteins and therefore the outgrowth rate. Regeneration ceases when the niche pool, and correspondingly Wnt and FGF production, depletes below an effective level. By this model, understanding instructive growth control mechanisms requires revealing niche cell origins and fates.

165 Regenerated fin tissues are derived from progenitor cells formed by partial dedifferentiation of mature cells extant at the injury site (Wehner and Weidinger, 2015). We sought to determine which pre-existing cell type(s) generates Dach $^{+}$niche cells. An obvious candidate was intra-ray mesenchyme, including joint-associated fibroblasts, that are activated some distance proximal to the amputation site and express tryptophan hydroxylase $1 b($ tph $1 b)$ (Tornini et al., 2016). These cells, which contribute to the distal blastema, are a source of replacement ray mesenchyme (Tornini et al., 2016; Tornini et al., 2017). We used a mosaic lineage tracing system (Stewart and Stankunas, 2012) to permanently label intra-ray mesenchyme of individual rays in uninjured fins by transgenic mCherry expression (Fig. 2A-D). We then amputated these fins and double stained 4 dpa sections containing labeled rays and derived regenerating tissue with Dach 175 and mCherry antibodies. Distal but not proximal mCherry-expressing blastema cells co- 
expressed Dach. Further, Dach ${ }^{+}$niche cells largely co-expressed Tg(tphlb:mCherry) (Fig. 2E, F). Finally, we found the fin regenerate-expressed Msx transcription factor (Smith et al., 2008) marks both proximal blastema cells and distal Dach-expressing cells (Fig. 2G). We conclude $\mathrm{Dach}^{+}$niche cells derive from intra-ray mesenchyme that initially populates the distal portion of the blastema.

The mesenchymal cell state marker Snail (Lamouille et al., 2014) was expressed in uninjured intra-ray and proximal regenerate mesenchymal cells (Fig. 2H, I). In contrast, Snail was absent from $\mathrm{Dach}^{+}$niche cells except for a small population of transitioning double $\mathrm{Dach}^{+} / \mathrm{Snail}^{+}$ cells (Fig. 2I). Concordantly, snai2 transcripts were abundant in blastema mesenchyme but 185 undetectable in distal-most tissue (Fig. S5). Dach-positive cells incorporated EdU even with a brief 4-hour pulse prior to tissue collection, showing the niche population includes actively proliferating cells (Fig. S5). These observations support a cyclical model whereby fin amputation induces a delayed transition of Snail ${ }^{+}$intra-ray mesenchyme to a $\mathrm{Dach}^{+}$niche state (Fig. 2J, K; Fig. S6). The Dach-expressing niche cells proliferate but, on net, progressively deplete through 190 their conversion back to Snail ${ }^{+}$mesenchyme that re-populates regenerated bony rays.

The origin of niche cells from intra-ray mesenchyme led us to consider that skeletal geometry might instruct how much niche is formed upon amputation. By this model, resecting a larger bony ray, with a relatively high volumetric capacity for mesenchyme, would produce a larger initial niche population and therefore more regeneration. In support, the size of fin ray 195 bone segments noticeably tapers along the proximal-to-distal axis (Fig. 3A-E). Further, longer peripheral rays are wider at their base than shorter central rays. 3-D micro-CT analysis confirmed this assessment and underscored that the internal geometry of the rays approximates cylinders (Fig. S7; Supplemental Video 1). Therefore, we considered the activated stretch of a 
ray extending proximally from an injury site (Knopf et al., 2011; Tornini et al., 2016) as a

200 cylinder of fixed length but variable width. As such, a ray's mesenchyme-holding capacity, injury-induced niche size, and finally fin outgrowth should correlate with ray widths at injury positions. We measured the width of the central sixteen rays at a fixed proximal-distal position and then plotted the radius ${ }^{\wedge} 2$ values as adjacent bars. The bars’ distribution approximated that of ray lengths, largely matching the overall shape of a zebrafish fin (Fig. 3A).

This model predicts niche amount should vary by proximal-distal amputation position, correlating with the tapering bony rays. Whole mount in situ hybridization for dachc transcripts at $3 \mathrm{dpa}$, the time of niche formation, revealed that proximal amputations produced large dachcexpressing niche pools while distal cuts generated progressively smaller niches (Fig. 3F-H). Further, dachc expression across the dorsal-to-ventral fin axis correlated with regenerative 210 demand upon perpendicular resections (Fig. S8) - as with diagonal amputations (Fig. 1H, I). Peripheral regions that re-form the longest rays expressed high levels of dachc. In contrast, central tissue that generates short rays to restore the original V-shaped fin produced small dachcmarked niches.

We used mathematical modeling to further explore if skeletal geometry coupled with 215 progressive niche depletion could account for robust size restoration during fin regeneration. We first assumed outgrowth rate is proportional to the current number of distal niche cells. We considered the volume of intra-ray mesenchyme activated proximally to an amputation site (Tornini et al., 2016) determines the size of the starting niche pool. As validated earlier, ray radius $^{\wedge} 2$ then serves as a proxy for the number of initial niche cells. A niche that collectively re220 differentiates faster than it proliferates over the course of regeneration establishes a "countdown timer". The number of initial niche cells sets the timer, with outgrowth ending when the niche 
population depletes below some effective level. As such, bony ray size at the amputation site ultimately instructs regenerated ray length.

We solved differential equations to derive a final equation describing regenerated ray 225 length as a function of ray radius at the amputation position and three "scaling parameters":

$$
l\left(t_{\text {end }}\right)=\frac{k_{l}}{\alpha}\left(N_{\Phi}-\beta \pi r^{2}\right)
$$

where $k_{/}$is the rate of length growth per niche cell, $\alpha$ is the difference in niche cell growth rate versus rate of differentiation back to intra-ray mesenchymal cells, $N_{\Phi}$ is the number of niche cells below which growth stops, and $\beta$ is the number of niche cells released per injury-activated ray volume. Mathematically exploring the formula reinforced the conclusion that $\alpha$ must be negative

230 to provide net niche depletion. Solving expected regenerated ray lengths using measured ray radii and optimized constants produced a distribution largely corresponding with actual measured lengths (Fig. 3I) - naturally matching normalized radius^2 distributions. A largely linear relationship between ray radius $^{\wedge} 2$ and remaining ray length from the measurement position held across the proximal-distal fin axis $($ Fig. 3J, R-squared $=0.7)$. Likewise, existent ray

235 lengths from a hypothetical injury point matched theoretical regenerated lengths derived from radius measurements and our growth equation (Fig. 3K).

Several zebrafish mutants develop and regenerate long fins, including dominant longfin ${ }^{t 2}$ (Van Eeden et al., 1996), well known as one of two mutations characterizing the Tüpfel longfin (TL) strain (Haffter et al., 1996) (Fig. 4A, B). longfin ${ }^{t 2}$ ray volumes estimated by normalized $240 \operatorname{radius}^{\wedge} 2$ measurements predicted overall fin shape but greatly under-anticipated ray lengths (Fig. 4C). Therefore, the skeleton was not proportionally re-scaled during development and instead suggesting longfin ${ }^{t 2}$ disrupts an outgrowth regulatory mechanism. We used our mathematical model to predict the growth-determining scaling parameter disrupted in longfin ${ }^{t 2}$ fish. The cell 
proliferation rate $\left(k_{l}\right)$ could be larger (e.g. increased mitogen production or mitogen sensitivity),

$245 \beta$ could be larger, establishing a larger niche pool upon injury, or $\alpha$ could be "less negative", leading to niche perdurance. Changing $k_{l}$ or $\beta$ vs. $\alpha$ had distinct theoretical effects when plotting expected regenerative growth over time and growth rate over time (Fig. 4D, E). Following amputation, longfin ${ }^{t 2}$ fish showed prolonged regenerative growth rather than increased initial growth or peak growth rate (Fig. 4F, G; Fig. S9), suggesting they have a persistent niche (less

250 negative $\alpha$ ) rather than enhanced niche generation $(\beta)$ or increased cellular growth rate $\left(k_{l}\right)$.

We examined dachc expression in longfin ${ }^{t 2}$ heterozygotes following fin resection to see if niche dynamics were disrupted as anticipated by our modeling (Fig. 4H-Q). longfin ${ }^{2}$ fish initially formed a normal-sized niche. However, unlike wildtype clutchmates, their dachc-expressing niche only slowly depleted, implicating a less negative $\alpha$ constant. Increasing $\alpha$ while inputting

255 longfin ${ }^{t 2}$ ray radii measurements largely predicted actual longfin ${ }^{t 2}$ regenerated ray lengths (Fig. S10). These results support the robustness of the model and show that longfin ${ }^{22}$ disrupts the niche depletion system.

We conclude ray volumes (set by their widths) are "transposed" into regenerated ray lengths, and hence fin form, via the amount of intra-ray mesenchyme exposed upon ray 260 resection. The mesenchyme transitions into a concordantly sized outgrowth-promoting niche pool that then steadily depletes, ultimately returning the regenerated fin to its original size and shape. In 1929, Samuel Nabrit similarly concluded: “... in [fin] regeneration the rate of growth and consequently the form is controlled locally by the cross-sectional area of the fin rays exposed" (Nabrit, 1929). Therefore, Nabrit's predicted growth promoting "fin ray end substance" 265 likely is the injury-released intra-ray mesenchyme itself. Positional information stored in tapering ray skeletal geometry is self-restoring since a depleting niche progressively directs 
slower outgrowth and narrower bones due to its diminishing progenitor-maintenance capacity. We use the term "transpositional scaling" to describe this model for organ size determination - a new answer to an age-old question of appendage regeneration.

Vertebrate appendage regeneration, including that in zebrafish fins, is used as compelling evidence for pattern formation by Cartesian coordinate-defined cellular positional identities (Wolpert, 2016). However, our transpositional scaling model does not require fin cells retain any such molecular information. Similarly, the hypothesized "pre-pattern" established early in a regenerating blastema that directs regional outgrowth (Nachtrab et al., 2013; Rabinowitz et al.,

275 2017; Rolland-Lagan et al., 2012; Tornini et al., 2016) can be explained by skeletal geometrydefined niche cell numbers alone. The state transition mechanisms that generate the niche and drive its progressive depletion are then identical throughout the fin but produce differential, predictable output depending on the starting condition defined by ray volumes.

Our conclusion linking longfin ${ }^{t 2}$ to disrupted niche depletion shows how a mutant can 280 have profound effects on fin morphology by altering the $\alpha$ scaling parameter defined by our mathematical model. As such, we provide a framework to understand evolutionary origins of appendage size and shape variation. In the accompanying manuscript, we identify cis ectopic expression of the voltage-gated potassium channel $k c n h 2 a$ in mesenchyme/niche lineage as the long sought cause of longfin ${ }^{22}$ (Stewart et al., 2019). These studies link ion signaling or 285 bioelectricity, widely connected to organ size control and regeneration (McLaughlin and Levin, 2018), to gene regulatory pathways regulating cell state transitions that slow then terminate fin outgrowth.

Could "transpositional scaling" models apply in other circumstances where an organ is restored to form and scale? The hemi-ray bones in zebrafish fins appose to approximate 
290 cylinders, conveniently enabling consideration of the geometric storage of a growth-promoting cell lineage. Urodele amphibian limb regeneration is another striking model of size and pattern restoration. Matching our idea, an older model proposes that initial blastema mass rather than origin determines which proximal-distal limb skeletal elements regenerate (de Both, 1970). However, urodele limb cartilage/bones are solid and therefore position-defining geometric

295 reservoirs of a hypothetical niche lineage would have to lie around rather than inside skeletal structures. Intriguingly, urodele limb positional information is stored in connective tissue mesenchyme surrounding bones, albeit reportedly by molecular memories (Kragl et al., 2009; McCusker et al., 2016; Nacu et al., 2013). Regardless, progenitor cell supporting-niche populations are a common component of organ growth, homeostasis, and regeneration (Chacón-

300 Martínez et al., 2018; Yamashita and Tumbar, 2014). Our simple model, inherently dependent on regulatory networks with the positional information stored in tissue geometry rather than molecular information, establishes a compelling paradigm to explore in many contexts. 


\section{MATERIALS AND METHODS}

\section{Zebrafish}

Wildtype AB (University of Oregon Zebrafish Facility), longfin ${ }^{t 2}$ (Haffter et al., 1996; Maderspacher and Nüsslein-Volhard, 2003; Van Eeden et al., 1996), Tg(-2.4shha:gfp:ABC) (Ertzer et al., 2007), $\operatorname{Tg}($ tphlb:mCherry) (Tornini et al., 2016), $\operatorname{Tg}($ dusp6:CreERT) (Stewart and Stankunas, 2012), and $\operatorname{Tg}(e a b: F l E x)$ (Boniface et al., 2009) zebrafish lines were used. Zebrafish

310 were housed in the University of Oregon Aquatic Animal Care Services facility at $28-29^{\circ} \mathrm{C}$. The University of Oregon Institutional Animal Care and Use Committee oversaw animal use.

\section{Transgenic mosaic fin lineage tracing}

Zebrafish embryos carrying the $\operatorname{Tg}(d u s p 6: C r e E R T)$ and $\operatorname{Tg}(e a b: F l E x)$ lines were treated with

315 low doses of tamoxifen, reared to adulthood, screened for mosaic labeling, caudal fin-amputated, and analyzed by whole mount imaging and antibody staining of sectioned tissue as described previously (Stewart and Stankunas, 2012).

\section{Small molecule studies of fin regeneration}

320 The Porcupine inhibitor Wnt-C59 (Proffitt et al., 2013), which blocks Wnt secretion, and the FGF receptor tyrosine kinase inhibitor PD173074 (Mohammadi et al., 1998) were dissolved and diluted in DMSO. Caudal fins were amputated at a fixed proximal-distal position or common oblique plane (illustrated in Fig. S1). Fin-resected fish were then immediately placed in drugcontaining aquarium water. Animals were transferred to fresh drug-containing water every 48

325 hours. The final concentration of DMSO in fish water was $0.01 \%$ for all conditions. 
For niche depletion experiments with delayed small molecule addition (Fig. S4), finresected $\mathrm{AB}$ wildtype fish first were allowed to regenerate for 4 days. The animals then were treated with DMSO $(0.01 \%, \mathrm{n}=10)$, Wnt-C59 (500 nM, n = 10), or PD173074 (5 $\mu \mathrm{M}, \mathrm{n}=10)$ for another 4 days. Animals were fed and changed to fresh drug-containing water after 48 hours.

330 At 8 days post-amputation (dpa), animals were returned to plain fish water. In some cases, fins were subjected to a second amputation proximal to the original amputation site at 12 dpa. Animals were followed for up to $60 \mathrm{dpa}$, when each fish was imaged using a Leica M165 FC stereomicroscope. Representative fish for each condition were visualized using high resolution stitched imaging (Nikon Eclipse Ti widefield microscope and NIS-Elements software) before 335 drug treatment, immediately after drug treatment, and at the experiment's conclusion. Regeneration was quantified by scoring the number of fully regenerated individual rays. This complete experiment was repeated three times with the same outcomes.

\section{RNA-Seq}

340 Caudal fins of adult $\operatorname{Tg}(-2.4$ shha:gfp:ABC) zebrafish were resected. At 4 dpa, dissected regenerated tissue at and beyond the distal GFP-marked epidermal domains was collected as “distal regenerate" samples. The remaining proximal regenerated tissue also was harvested. Tissue pooled from four animals constituted matched replicate samples. Tissue was immediately placed in TRIzol reagent (Thermo Fisher) and homogenized. RNA was isolated following the

345 manufacturer's instructions with minor alterations. The RNA was precipitated overnight at $-80^{\circ} \mathrm{C}$ and then pelleted at $15,000 \mathrm{rpm}$ for 30 minutes at $4^{\circ} \mathrm{C}$. Pellets were washed twice with $70 \%$ ethanol, dried for 10 minutes at room temperature, and resuspended in RNase-free water (Thermo Fisher). 
RNA-Seq libraries were prepared from $1 \mu \mathrm{g}$ of isolated RNA using a Kapa Biosystems

350 Stranded mRNA-Seq kit. Bar-coded libraries were pooled and sequenced using a NextSeq 500 (Illumina). Illumina reads were aligned to the zebrafish genome (GRCz11) using TopHat2 (Kim et al., 2013). Gene-assigned aligned reads were counted using HTseq (Anders et al., 2015). DEseq (Anders and Huber, 2010) was used to determine differential expression of 1147 genes annotated to have "transcription regulator activity" (GO:0140110) by AmiGO 2 (Ashburner et

355 al., 2000; Carbon et al., 2009; Gene Ontology Consortium et al., 2013). Genes below the 40\% quantile for total counts were filtered out to improve the statistical analysis of relatively highly expressed genes.

\section{Immunostaining and in situ hybridization}

360 Immunostaining was performed on paraffin sections as described (Stewart et al., 2014). Antibodies used were: Runx2 (Santa Cruz Biotechnology, 27-K, 1:500-1000 dilution); Dach (Proteintech Group, 1:2000); Msx (Developmental Studies Hybridoma Bank, tissue culture supernatant, 1:15-20); Snail (Developmental Studies Hybridoma Bank, ascites, 1:100); mCherry (Takara Bio or Novus Biologicals, 1:100). For Snail/Dach double immunostaining, Dach

365 antibodies were used at 1:1000 and sodium dodecyl sulfate (SDS) was added to 0.005\% during the primary antibody incubation step. Immunostained sections were imaged using either Olympus FV1000 or Zeiss LSM 880 laser scanning confocal microscopes.

Whole mount in situ hybridization using DIG-labeled probes followed established procedures (Stewart et al., 2014). The wnt5a probe was described previously (Stewart et al., 370 2014). Templates for dacha and dachc probes were amplified by PCR from regenerating fin cDNA using the indicated primers, where the reverse primer contains a $\mathrm{T} 7$ promoter sequence 
(in caps) for in vitro transcription using DIG labeling mixtures (Roche) and T7 RNA polymerase:

dacha forward: 5'-atggecgtatctgcaactcctccggtgc-3' dacha reverse: 5'-TAATACGACTCACTATAGGGtcagtacatgatggggggtttggagtagg-3' dachc forward: 5'-atggccacgcgectccga-3' dachc reverse: 5'-TAATACGACTCACTATAGGGtcagtacatcattgtggactttagaaagagcctt-3' The snai2 probe template was generated by PCR amplifying its coding sequence from regenerating fin cDNA using the following primers followed by ligation to the pCRII vector 380 (Thermo Fisher).

snai2 forward: 5'-atgcctcgttcattcctagtaaagaagc-3' snai2 reverse: 5'-tcagtgtgcgatgcaacagccag-3'

The resulting plasmid was linearized and a DIG-labeled probe synthesized by in vitro transcription using SP6 RNA polymerase.

\section{5-ethynyl-2'-deoxyuridine (EdU) incorporation and staining}

$10 \mu \mathrm{g}$ of EdU in saline was intraperitoneally injected into wildtype fish 92 hours post caudal fin amputation. Fin tissue was collected 4 hours later, fixed, and processed for paraffin sectioning. EdU detection used a Click-iT kit (Thermo Fisher) combined with immunostaining as described 390 above using Dach antibodies.

\section{X-ray micro computed-tomography imaging}

An adult wildtype zebrafish was sacrificed and fixed in 4\% PFA for 24 hours. The specimen was stabilized within a $15 \mathrm{ml}$ conical tube and its caudal fin scanned using a VivaCT 80 (SCANCO 
395 Medical) with $55 \mathrm{kVp}$ X-ray source energy, $145 \mu \mathrm{A}$ current, $6.5 \mu \mathrm{m}$ pixel size and $1000 \mathrm{~ms}$ per projection integration time. Slice images were reconstructed to a $1590 \times 1590$ pixel matrix using an automated cone beam convolution backprojection algorithm. The resulting 2-D images were output for 3-D reconstruction using Imaris 9.3 software (Bitplane).

\section{Morphometrics and mathematical modeling}

Rays were considered as simple cylinders to facilitate modeling regenerative fin outgrowth as a function of skeletal geometry. A given bony ray segment's volumetric capacity for injuryactivated, niche-forming intra-ray mesenchyme (Tornini et al., 2016), then could be estimated by first measuring the ray's width (= diameter) at a hypothetical amputation site. For modeling

405 purposes, the square of the ray's radius was used as a proxy as normalized areas and volumes (for cylinders of a fixed length) are mathematically identical.

Caudal fins of five-month-old longfin ${ }^{t 2 /+}$ and wildtype clutchmate fish were imaged using differential interference contrast microscopy and multi-field stitching (Nikon Eclipse Ti and NIS-Elements) for morphometrics. Fish were euthanized by tricaine bath overdose and mounted 410 in $0.75 \%$ low melt agarose / PBS on microscope slides. The fins were gently fanned out using a horse hair brush to fully extend the tissue and separate rays. The initial proximal-distal positions for ray measurements were determined by first identifying the end of the innermost truncated rays (peripheral "mini-rays" that, unlike the standard 18 caudal rays, do not extend the full distance of the fin, shown in Fig. S1). A line was drawn across the fin that intersected points two

415 ray bone segments preceding the end of the mini-rays on the fin's dorsal and ventral sides. The width of each ray was measured where it intersected this transverse line. 
Similarly, polylines (using anchored points to allow for shifts in ray orientation) starting from the same proximal-distal position were drawn along each ray and then directly in between branches of a given "mother ray" until reaching the fin tip. The lengths of these lines defined 420 how much each ray would have to regenerate to restore the fin's original size. Lengths were normalized to the longest wildtype ray. Repeated measures taken every three bone segments along the proximal-distal axis for rays 3, 4, 15, 16 used the same procedure, with lengths representing the distance from the diameter measurement position to the end of the ray.

We derived a mathematical model for "Transposition Scaling" that predicts the extent of 425 regenerative outgrowth as a function of skeletal geometry - the bony ray's capacity for nicheoriginating mesenchyme. First, we considered the number of niche cells released at a cut site as:

$$
N_{0} \equiv \beta \pi r^{2}
$$

where $r$ is the radius of the ray at the cut site and $\beta$ is the average number of niche cells released per unit area. Niche cells can then proliferate (given by $k_{g}$ ) or convert to a differentiated, mesenchymal state (given by $k_{q}$ ). The change in the number of niche cells per unit time is:

$$
\frac{d N}{d t}=\left(k_{g}-k_{q}\right) N(t)
$$

430 The difference in these rates can be summarized by $\alpha \equiv\left(k_{g}-k_{q}\right)$. Using the boundary condition that $N(0)=N_{0}$, the solution to this first-order differential equation is:

$$
N(t)=\beta \pi r^{2} e^{\alpha t}
$$

We model bony ray growth rate as directly proportional to the number of niche cells, controlled by $k_{l}$ :

$$
\frac{d l}{d t}=k_{l} N(t)=k_{l} \beta \pi r^{2} e^{\alpha t}
$$

Using the boundary condition that $l(0)=0$, the solution to this differential equation is: 


$$
l(t)=\frac{k_{l} \beta \pi r^{2}}{\alpha}\left(e^{\alpha t}-1\right)
$$

435 To link the number of cells released to the final ray length, we assume that skeletal growth stops when the number of cells reaches $N_{\Phi}$, the minimum number of cells required to support growth. This happens at $t_{\text {end }}$ :

$$
N_{\Phi}=\beta \pi r^{2} e^{\alpha t_{e n d}}
$$

We then solve for $t_{\text {end }}$ :

$$
t_{\text {end }}=\frac{\ln \left(\frac{N_{\Phi}}{\beta \pi r^{2}}\right)}{\alpha}
$$

and substitute into our model for $l(t)$ :

$$
\begin{gathered}
l\left(t_{\text {end }}\right)=\frac{k_{l} \beta \pi r^{2}}{\alpha}\left(e^{\alpha \frac{\ln \left(\frac{N_{\Phi}}{\beta \pi r^{2}}\right)}{\alpha}}-1\right) \\
l\left(t_{\text {end }}\right)=\frac{k_{l} \beta \pi r^{2}}{\alpha}\left(\frac{N_{\Phi}}{\beta \pi r^{2}}-1\right)
\end{gathered}
$$

440 This gives the final relationship:

$$
l\left(t_{\text {end }}\right)=\frac{k_{l}}{\alpha}\left(N_{\Phi}-\beta \pi r^{2}\right)
$$

where $k_{l}$ is the length growth rate per niche cell, $\alpha$ is the difference between the niche cell growth rate and their conversion back to intra-ray mesenchymal cells, $N_{\Phi}$ is the minimum number of cells required to support growth, and $\beta$ reflects how many niche cells are released per bony ray cross-sectional area.

\section{Fin regeneration outgrowth measurements}

Caudal fins from clutchmate longfin ${ }^{+/+}$and longfin ${ }^{\text {t2/+ }}$ animals were amputated at the same position along the proximal-distal axis (Fig. S1) and allowed to regenerate for 30 days. 
Outgrowth measurements were taken every 24 hours from 1-8 dpa and every 48 hours from 10-

45030 dpa. The length of the third ray (Fig. S1) from the amputation site to the fin's tip was measured from images acquired with a Leica M205 FA stereomicroscope. Stitched fin images of zebrafish euthanized with tricaine and mounted in $0.75 \%$ low melting agarose were captured using a Nikon Eclipse Ti widefield microscope and NIS-Elements software. Data points were fit to user-defined transpositional scaling equations (see Mathematical modeling) by nonlinear

455 regression using GraphPad Prism. The $\alpha, \beta$, and $k_{l}$ parameters were allowed to vary to generate best-fit curves. $\beta$ and $k_{l}$ were nearly identical between the wildtype and longfin ${ }^{t 2 /+}$ data sets. In contrast, $\alpha$ was 3.0x higher for longfin ${ }^{t 2 /+}$, similar to the $\alpha$ difference $(2.1 \mathrm{x})$ derived from the geometrical (rather than growth rate) analysis. 


\section{ACKNOWLEDGEMENTS}

We thank C. Kimmel and V. Devasthali for feedback on the manuscript; the Stankunas lab for discussions; E. Shaw for technical assistance; the University of Oregon X-ray Imaging Core and A. Lin for assisting with micro-CT; K. Poss and ZIRC for providing zebrafish lines; and the DSHB for antibodies.

\section{FOOTNOTES}

\section{Competing interests}

None.

\section{Author contributions}

470 S. S., G. A. Y., H. K. L. and K. S. designed experiments. S.S., G. A. Y., H. K. L., A. L. H., J. A. B., and J. C. performed experiments. K. S. and M. J. H. led the mathematical modeling. K. S. and S. S. prepared and wrote the manuscript with input from G. A. Y., H. K. L., M. J. H., and A. L. H.

\section{Funding}

475 The National Institutes of Health (NIH) provided research funding (1R01GM127761 (K. S. and S. S.) and 5R03AR067522 (S. S.)). G. A. Y. was supported by a NIH NRSA graduate fellowship (5F31AR071283). H. K. L. received support from the University of Oregon Developmental Biology Training Program (5T32HD007348).

\section{Data and material availability} materials should be addressed to S. S. and K. S. 


\section{REFERENCES}

Anders, S. and Huber, W. (2010). Differential expression analysis for sequence count data. Genome Biol. 11, R106.

Anders, S., Pyl, P. T. and Huber, W. (2015). HTSeq--a Python framework to work with highthroughput sequencing data. Bioinformatics 31, 166-169.

Ashburner, M., Ball, C. A., Blake, J. A., Botstein, D., Butler, H., Cherry, J. M., Davis, A. P., Dolinski, K., Dwight, S. S., Eppig, J. T., et al. (2000). Gene ontology: tool for the unification of biology. The Gene Ontology Consortium. Nature Genetics 25, 25-29.

Boniface, E. J., Lu, J., Vicotroff, T., Zhu, M. and Chen, W. (2009). FlEx-based transgenic reporter lines for visualization of Cre and Flp activity in live zebrafish. Genesis (New York, N.Y. : 2000) 47, 484-491.

Broussonet, M. (1786). Observations sur la régénération de quelques parties du corps des poissons. Histoire de lAcadémie Royale des Sciences 684-688.

Carbon, S., Ireland, A., Mungall, C. J., Shu, S., Marshall, B., Lewis, S., AmiGO HubWeb Presence Working Group (2009). AmiGO: online access to ontology and annotation data. Bioinformatics 25, 288-289.

Chacón-Martínez, C. A., Koester, J. and Wickström, S. A. (2018). Signaling in the stem cell niche: regulating cell fate, function and plasticity. Development 145, dev165399.

de Both, N. J. (1970). The developmental potencies of the regeneration blastema of the axolotl limb. Wilhelm Roux Arch Entwickl Mech Org 165, 242-276.

Ertzer, R., Müller, F., Hadzhiev, Y., Rathnam, S., Fischer, N., Rastegar, S. and Strähle, U. (2007). Cooperation of sonic hedgehog enhancers in midline expression. Developmental Biology 301, 578-589.

505 Gene Ontology Consortium, Blake, J. A., Dolan, M., Drabkin, H., Hill, D. P., Li, N., Sitnikov, D., Bridges, S., Burgess, S., Buza, T., et al. (2013). Gene Ontology annotations and resources. Nucleic Acids Res. 41, D530-5.

Haffter, P., Odenthal, J., Mullins, M. C., Lin, S., Farrell, M. J., Vogelsang, E., Haas, F., Brand, M., Van Eeden, F. J., Furutani-Seiki, M., et al. (1996). Mutations affecting pigmentation and shape of the adult zebrafish. Dev. Genes Evol. 206, 260-276.

Kim, D., Pertea, G., Trapnell, C., Pimentel, H., Kelley, R. and Salzberg, S. L. (2013). TopHat2: accurate alignment of transcriptomes in the presence of insertions, deletions and gene fusions. Genome Biol. 14, R36.

Knopf, F., Hammond, C., Chekuru, A., Kurth, T., Hans, S., Weber, C. W., Mahatma, G., Fisher, S., Brand, M., Schulte-Merker, S., et al. (2011). Bone Regenerates via Dedifferentiation of Osteoblasts in the Zebrafish Fin. Developmental Cell 20, 713-724. 
Kragl, M., Knapp, D., Nacu, E., Khattak, S., Maden, M., Epperlein, H. H. and Tanaka, E. M. (2009). Cells keep a memory of their tissue origin during axolotl limb regeneration. Nature 460, 60-65.

Lamouille, S., Xu, J. and Derynck, R. (2014). Molecular mechanisms of epithelialmesenchymal transition. Nat Rev Mol Cell Biol 15, 178-196.

Lee, Y., Grill, S., Sanchez, A., Murphy-Ryan, M. and Poss, K. D. (2005). Fgf signaling instructs position-dependent growth rate during zebrafish fin regeneration. Development 132, 5173-5183.

525 Lee, Y., Hami, D., De Val, S., Kagermeier-Schenk, B., Wills, A. A., Black, B. L., Weidinger, G. and Poss, K. D. (2009). Maintenance of blastemal proliferation by functionally diverse epidermis in regenerating zebrafish fins. Developmental Biology 331, 270-280.

Maderspacher, F. and Nüsslein-Volhard, C. (2003). Formation of the adult pigment pattern in zebrafish requires leopard and obelix dependent cell interactions. Development 130, 34473457.

Mardon, G., Solomon, N. M. and Rubin, G. M. (1994). dachshund encodes a nuclear protein required for normal eye and leg development in Drosophila. Development 120, 3473-3486.

McCusker, C. D., Diaz-Castillo, C., Sosnik, J., Q Phan, A. and Gardiner, D. M. (2016). Cartilage and bone cells do not participate in skeletal regeneration in Ambystoma mexicanum limbs. Developmental Biology 416, 26-33.

McLaughlin, K. A. and Levin, M. (2018). Bioelectric signaling in regeneration: Mechanisms of ionic controls of growth and form. Developmental Biology 433, 177-189.

Mohammadi, M., Froum, S., Hamby, J. M., Schroeder, M. C., Panek, R. L., Lu, G. H., Eliseenkova, A. V., Green, D., Schlessinger, J. and Hubbard, S. R. (1998). Crystal structure of an angiogenesis inhibitor bound to the FGF receptor tyrosine kinase domain. EMBO J. 17, 5896-5904.

Morgan, T. H. (1900). Regeneration in teleosts. Archiv für Entwicklungsmechanik der Organismen 10, 120-134.

Morrison, S. J. and Spradling, A. C. (2008). Stem cells and niches: mechanisms that promote stem cell maintenance throughout life. Cell 132, 598-611.

Nabrit, S. M. (1929). The role of the fin rays in the regeneration in the tail-fins of fishes. Biological Bulletin LVI, 235-266.

Nachtrab, G., Kikuchi, K., Tornini, V. A. and Poss, K. D. (2013). Transcriptional components of anteroposterior positional information during zebrafish fin regeneration. Development 140, 3754-3764. 
Nacu, E., Glausch, M., Le, H. Q., Damanik, F. F. R., Schuez, M., Knapp, D., Khattak, S., Richter, T. and Tanaka, E. M. (2013). Connective tissue cells, but not muscle cells, are involved in establishing the proximo-distal outcome of limb regeneration in the axolotl. Development 140, 513-518.

Nechiporuk, A. and Keating, M. T. (2002). A proliferation gradient between proximal and msxb-expressing distal blastema directs zebrafish fin regeneration. Development 129, 2607 2617.

Poss, K. D., Shen, J., Nechiporuk, A., McMahon, G., Thisse, B., Thisse, C. and Keating, M. T. (2000). Roles for Fgf signaling during zebrafish fin regeneration. Developmental Biology 222, 347-358.

Proffitt, K. D., Madan, B., Ke, Z., Pendharkar, V., Ding, L., Lee, M. A., Hannoush, R. N. and Virshup, D. M. (2013). Pharmacological inhibition of the Wnt acyltransferase PORCN prevents growth of WNT-driven mammary cancer. Cancer Res. 73, 502-507.

Rabinowitz, J. S., Robitaille, A. M., Wang, Y., Ray, C. A., Thummel, R., Gu, H., Djukovic, D., Raftery, D., Berndt, J. D. and Moon, R. T. (2017). Transcriptomic, proteomic, and metabolomic landscape of positional memory in the caudal fin of zebrafish. Proc. Natl. Acad. Sci. U.S.A. 114, E717-E726.

Rolland-Lagan, A.-G., Paquette, M., Tweedle, V. and Akimenko, M.-A. (2012). Morphogenbased simulation model of ray growth and joint patterning during fin development and regeneration. Development 139, 1188-1197.

Shen, W. and Mardon, G. (1997). Ectopic eye development in Drosophila induced by directed dachshund expression. Development 124, 45-52.

Shibata, E., Yokota, Y., Horita, N., Kudo, A., Abe, G., Kawakami, K. and Kawakami, A. (2016). Fgf signalling controls diverse aspects of fin regeneration. Development 143, 2920 5752929.

Smith, A., Zhang, J., Guay, D., Quint, E., Johnson, A. and Akimenko, M. A. (2008). Gene expression analysis on sections of zebrafish regenerating fins reveals limitations in the whole-mount in situ hybridization method. Dev. Dyn. 237, 417-425.

Stewart, S. and Stankunas, K. (2012). Limited dedifferentiation provides replacement tissue during zebrafish fin regeneration. Developmental Biology 365, 339-349.

Stewart, S., Gomez, A. W., Armstrong, B. E., Henner, A. and Stankunas, K. (2014). Sequential and opposing activities of Wnt and BMP coordinate zebrafish bone regeneration. Cell Reports 6, 482-498.

Stewart, S., Le Bleu, H. K., Yette, G. A., Henner, A. L., Braunstein, J. A. and Stankunas, K. (2019). longfin causes cis-ectopic expression of the kcnh2a ether-a-go-go $\mathrm{K}^{+}$channel to autonomously prolong fin outgrowth. bioRxiv 790329. 
Stoick-Cooper, C. L., Weidinger, G., Riehle, K. J., Hubbert, C., Major, M. B., Fausto, N. and Moon, R. T. (2007). Distinct Wnt signaling pathways have opposing roles in appendage regeneration. Development 134, 479-489.

Tornini, V. A., Puliafito, A., Slota, L. A., Thompson, J. D., Nachtrab, G., Kaushik, A.-L., Kapsimali, M., Primo, L., Di Talia, S. and Poss, K. D. (2016). Live Monitoring of Blastemal Cell Contributions during Appendage Regeneration. Curr. Biol. 26, 2981-2991.

Tornini, V. A., Thompson, J. D., Allen, R. L. and Poss, K. D. (2017). Live fate-mapping of joint-associated fibroblasts visualizes expansion of cell contributions during zebrafish fin regeneration. Development 144, 2889-2895.

Van Eeden, F. J., Granato, M., Schach, U., Brand, M., Furutani-Seiki, M., Haffter, P., Hammerschmidt, M., Heisenberg, C. P., Jiang, Y. J., Kane, D. A., et al. (1996). Genetic analysis of fin formation in the zebrafish, Danio rerio. Development 123, 255-262.

Wehner, D. and Weidinger, G. (2015). Signaling networks organizing regenerative growth of the zebrafish fin. Trends Genet. 31, 336-343.

Wehner, D., Cizelsky, W., Vasudevaro, M. D., Özhan, G., Haase, C., Kagermeier-Schenk, B., Röder, A., Dorsky, R. I., Moro, E., Argenton, F., et al. (2014). Wnt/ $\beta$-catenin signaling defines organizing centers that orchestrate growth and differentiation of the regenerating zebrafish caudal fin. Cell Reports 6, 467-481.

605 Wolpert, L. (2016). Positional Information and Pattern Formation. Curr. Top. Dev. Biol. 117, 597-608.

Yamashita, Y. M. and Tumbar, T. (2014). Stem cells and their niche in homeostasis/regeneration and disease. Mol. Biol. Cell 25, 736-736. 


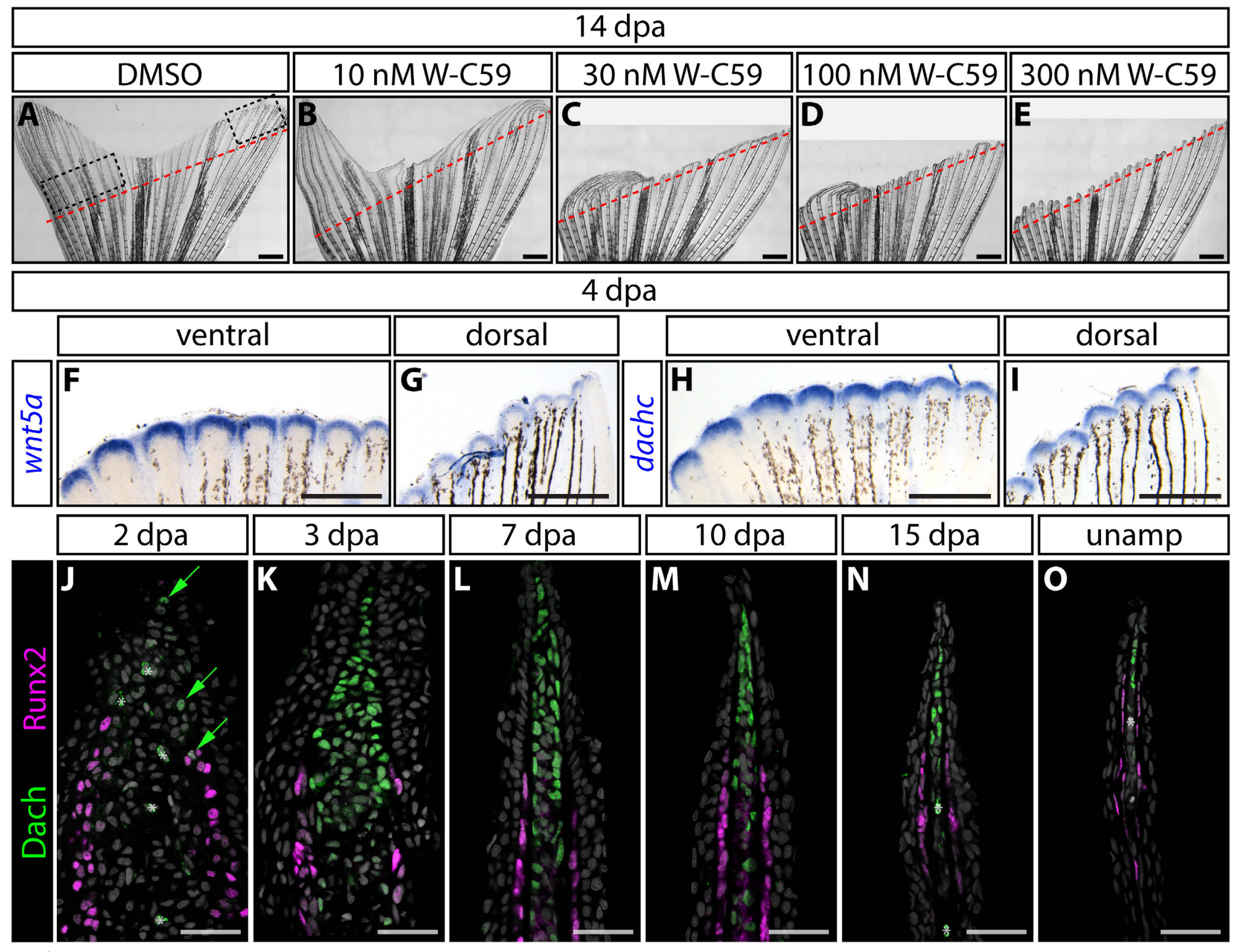

Figure 1 
Figure 1. Dachshund transcription factors define a progressively depleting pool of distal niche cells in regenerating fins.

(A-E) Sensitivity to Wnt inhibition inversely correlates with demand for regenerative growth.

615 Diagonally fin-amputated zebrafish exposed to increasing concentrations of Wnt-C59 Porcupine inhibitor and imaged at 14 days post amputation (dpa). Red dashed lines show the amputation plane. Scale bars are $1 \mathrm{~mm}$. (F-I) The size of distal wnt5a and dachc expression domains positively correlate with regenerative demand following diagonal amputations. Images compare whole mount RNA in situ hybridization signal intensities between ventral (proximally 620 amputated) and dorsal (distally amputated) sites along the diagonal resection plane, approximated by dashed boxes in (A). Scale bars are $500 \mu \mathrm{m}$. (J-O) Dachshund (Dach) expression defines "niche" cells in the distal blastema, distinct from adjacent Runx2-expressing progenitor osteoblasts. Dach $^{+}$cells are first observed at 2 dpa, peak in number around 3 dpa, and then progressively decrease to a small residual pool that is also present in unamputated fins.

625 Images show antibody-stained regenerating fin sections at the indicated times post-amputation. Dach and Runx2 are green and magenta, respectively. Hoechst-stained nuclei are grey. Asterisks denote red blood cells. Scale bars are $25 \mu \mathrm{m}$. 


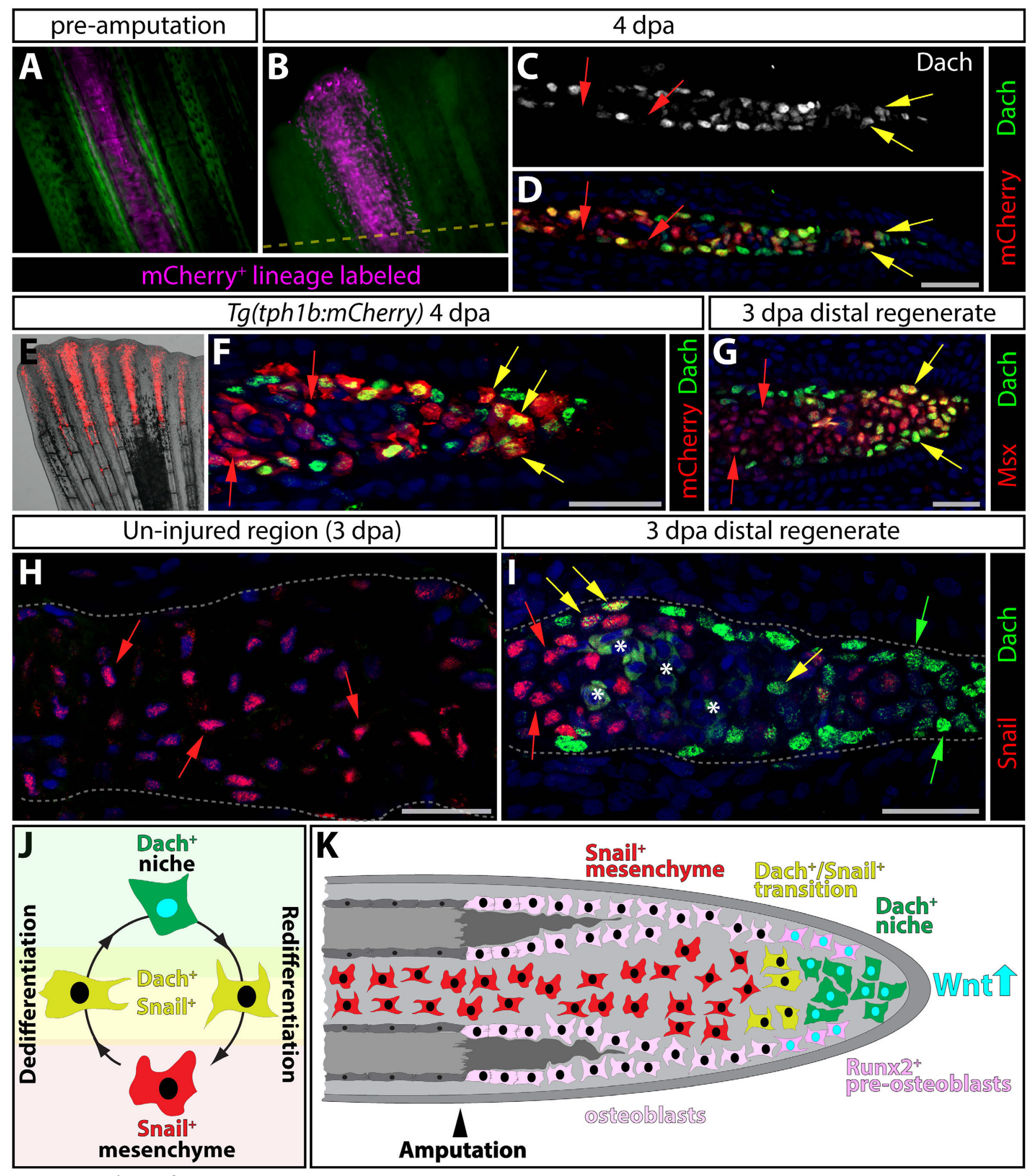

Figure 2 


\section{Figure 2. Niche cells are generated from state-transitioning intra-ray mesenchyme.}

630 (A-D) Genetic mosaic analysis demonstrates that the Wnt-producing niche derives from intra-ray mesenchyme. Example of a genetic mosaic fin with a single ray containing mCherry-labeled mesenchyme (in magenta) prior to amputation (A) and 4 dpa (B). The amputation plane is indicated with a dashed yellow line. (C, D) A section of the same ray at 4 dpa stained with antiDach (grey in single channel image in C, green in D) and anti-mCherry antibodies (red). Red arrows mark mCherry ${ }^{+}$but Dach ${ }^{-}$blastema cells and yellow arrows highlight $\mathrm{Dach}^{+} / \mathrm{mCherry}^{+}$ niche cells. (E, F) $\operatorname{Tg}(t p h 1 b:$ Cherry) expression highlights the regenerating intra-ray mesenchyme/niche lineage. Immunostaining of a 4 dpa $\operatorname{Tg}($ tph $1 b: m C h e r r y)$ fin section shows Dach (green) and tph1b (red) expressing niche cells (yellow arrows). Red arrows mark further proximal Dach $/ \mathrm{mCherry}^{+}$blastema cells. (G-I) Mesenchymal-to-niche cell transitions at 4 dpa

640 defined by Dach, Msx, and Snail transcription factors. (G) Like tph1b:mCherry, Msx expression (red) labels the lineage whether in a differentiated mesenchyme (red arrows) or Dach-expressing (green) niche (yellow arrows) state. (H, I) Snail expression (red nuclei shown with red arrows) marks intra-ray mesenchyme proximal to the amputation site and blastema cells except distal $\mathrm{Dach}^{+}$niche cells (green nuclei highlighted by green arrows). A small number of Snail ${ }^{+} / \mathrm{Dach}^{+}$

645 cells (yellow nuclei indicated by yellow arrows) identify cells transitioning between mesenchyme and niche states. Scale bars in all panels are $25 \mu \mathrm{m}$. Asterisks denote red blood cells. (J). Model of cell state transitions between intra-ray mesenchyme and niche cells during regeneration. Snail ${ }^{+}$mesenchyme cells normally populate the intra-ray space. After amputation, Snail $^{+}$cells dedifferentiate into $\mathrm{Dach}^{+}$niche cells. As ray outgrowth proceeds, niche cells 650 redifferentiate to contribute Snail ${ }^{+}$intra-ray mesenchyme. (K) Control of regeneration by a Wntproducing dynamic niche pool. Runx $2^{+}$osteoblasts (in pink) are maintained by Wnt (represented 
bioRxiv preprint doi: https://doi.org/10.1101/606970; this version posted October 2, 2019. The copyright holder for this preprint (which was not certified by peer review) is the author/funder. All rights reserved. No reuse allowed without permission.

by cyan nuclei) secreted by the distal $\mathrm{Dach}^{+} / \mathrm{Wnt}^{+}$niche (green cells with cyan nuclei), which transition (yellow cells) back to Snail ${ }^{+}$mesenchyme (red cells) as regeneration progresses. 

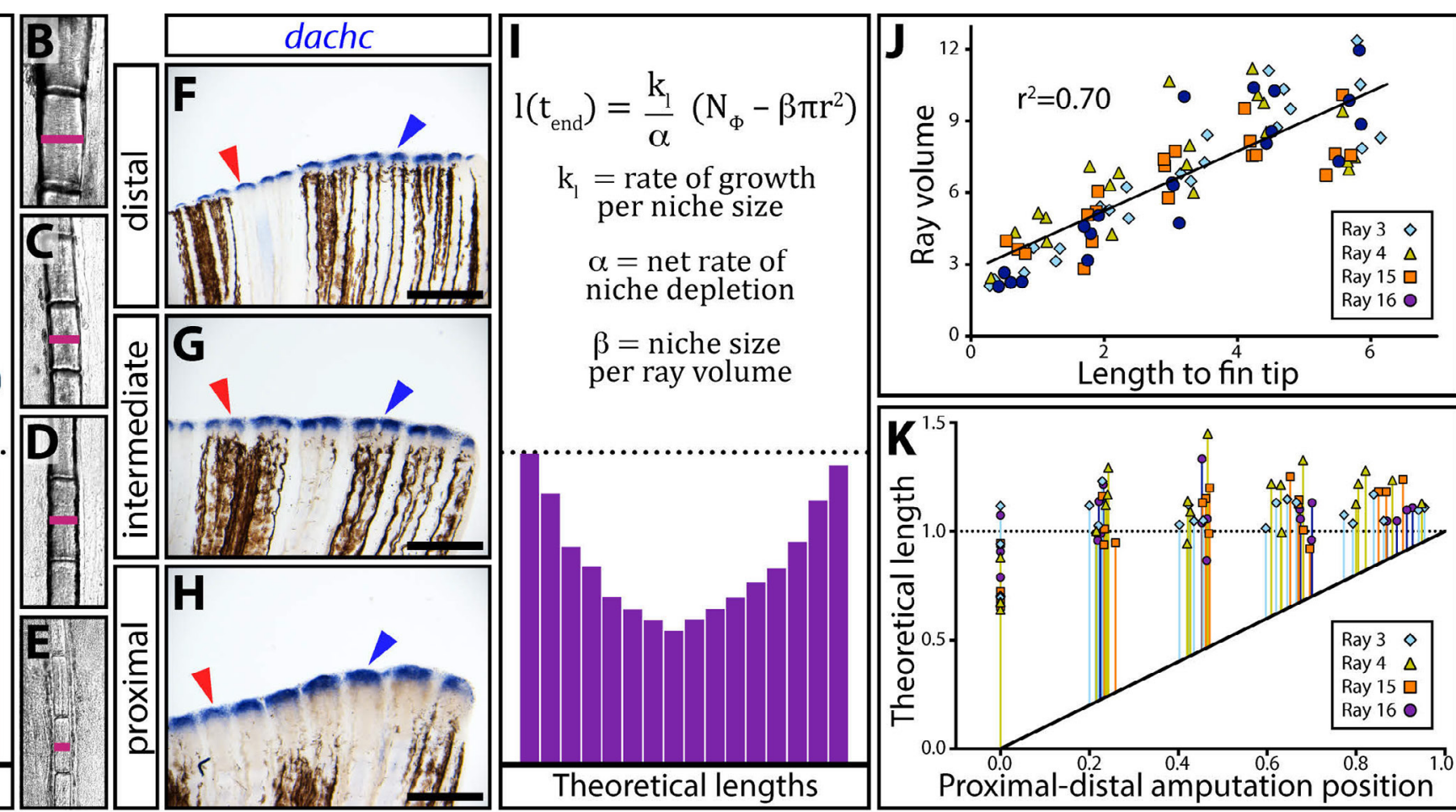

Figure 3 


\section{Figure 3. Skeletal geometry-dependent niche generation with progressive niche depletion} models regenerated ray length.

(A) A ray's cross-sectional area (therefore, volume) exposed upon amputation (modeled by the square of the radius, $r^{2}$, pink) anticipates the length (l, light blue) it will regenerate. Plotted normalized measurements for the sixteen central rays show a tight correlation between ray

660 lengths and ray volumes, from/at a hypothetical proximal amputation position (green line). Error bars are one standard deviation $(n=4)$. (B-E) Scale-matched zoom images of the ray segments boxed in the stitched whole fin image in (A), showing differential girth depending on bone position. Pink lines mark ray diameters. (F-H) dachc whole mount in situ hybridizations on 72 hpa regenerating fins amputated at distal $(\mathrm{F})$, intermediate $(\mathrm{G})$, or proximal $(\mathrm{H})$ positions. As

665 anticipated by the model, dachc expression, representing the amount of niche generated, correlates with regenerative demand. Scale bars are $500 \mu \mathrm{m}$. (I) A mathematical model equation represents the length of a given ray at the end of regeneration $\left(l\left(t_{\text {end }}\right)\right)$ as a function of the radius of the ray at the amputation position. Three scaling parameters $\left(k_{l}, \alpha\right.$, and $\left.\beta\right)$ must be tuned precisely to restore the original length, but no molecular positional information is required. The

670 plot shows how the formula theoretically restores normalized ray lengths using actual measured radius values at the hypothetical amputation point (green line). (J) Scatter plot showing a linear relationship between ray volume and ray length from any measured position along the proximaldistal (P-D) fin axis. Each point represents 5 or 6 measured points from each of rays 3, 4, 15, 16 from four animals. Matching data point colors/shapes indicates a given ray. Units are arbitrary.

675 (K) The mathematical model predicts regenerated length (normalized $=1$ ) regardless of amputation position (normalized units). Each data point shows the theoretical length derived from a given ray's radius measurements at a single P-D position. The colored vertical lines 
bioRxiv preprint doi: https://doi.org/10.1101/606970; this version posted October 2, 2019. The copyright holder for this preprint (which was not certified by peer review) is the author/funder. All rights reserved. No reuse allowed without permission.

represent anticipated growth from the amputation position (therefore, starting from the black diagonal line). 


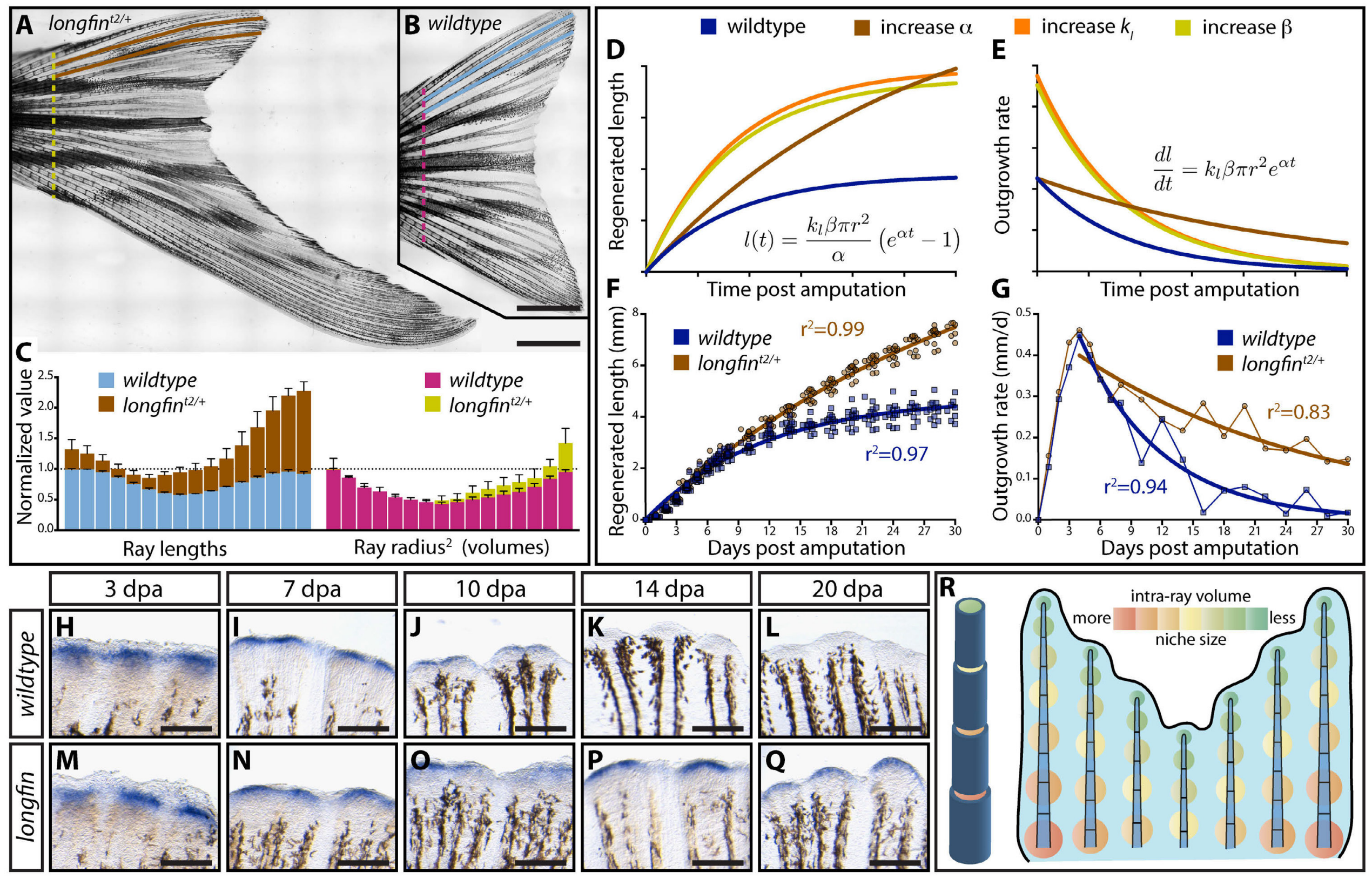

Figure 4 


\section{Figure 4. longfin fish regenerate exceptionally long fins due to a broken niche countdown} timer, supporting a transpositional scaling model of self-restoring fin geometry.

(A, B) Stitched differential interference contrast images show the dramatically overgrown caudal fins of longfin ${ }^{t 2 /+}$ fish. Ray length and radius measurements taken at/from a proximal position denoted by colored dashed lines (brown/yellow=longfin ${ }^{t 2 /+}$, light blue/pink=wildtype). Scale bars are $2 \mathrm{~mm}$. (C) Normalized caudal fin ray lengths (left) and volumes (from radius $^{2}$ measurements, right) for clutchmate wildtype and longfin ${ }^{t 2 /+}$ fish. (D) Theoretical regeneration growth and (E) growth rate curves derived from the transpositional scaling equation have distinct shapes depending if niche response $\left(k_{l}\right)$ or niche generation $(\beta)$ vs. niche perdurance $(\alpha)$ parameters are

690 increased. The actual growth $(\mathbf{F})$ and growth rate $(\mathbf{G})$ of regenerating longfin ${ }^{t 2 /+}$ caudal fins match expectations if niche perdurance $(\alpha)$ - the "countdown timer" - is disrupted. The initial and maximum growth rate is unaltered but the growth rate progressively decreases at a much slower rate. Curves show actual data fit to the modeling equations (r-squared goodness of fit values are shown). All data points ( $\geq 12$ fish per time) are shown in $(\mathrm{F})$; points in $(\mathrm{G})$ are mean

695 values. (H-Q) Whole mount in situ hybridization for the dachc niche marker through a time course post-caudal fin amputation for wildtype and longfin ${ }^{t 2 /+}$ fish. longfin ${ }^{t 2 /+}$ fish generate a normal-sized niche after fin resection but the cells fail to deplete in a timely manner. Scale bars are $200 \mu \mathrm{m}$. (R) A "Transpositional Scaling" model can explain how positional information stored in skeletal geometry combined with a state transitioning and depleting niche population

700 directs a fin to regenerate back to its original size and shape. A wider amputated ray produces more niche cells from its intra-ray mesenchyme (A tapered, segmented ray is shown on the left, with intra-ray volume graded from orange (more) to green (less)). Niche cells deplete at a constant rate through their re-differentiation to mesenchyme (starting and then diminishing niche 
bioRxiv preprint doi: https://doi.org/10.1101/606970; this version posted October 2, 2019. The copyright holder for this preprint (which was not certified by peer review) is the author/funder. All rights reserved. No reuse allowed without permission.

pools represented by same orange-to-green gradation as well as circle sizes on the right fin

705 graphic). The diminishing niches maintain progressively smaller bone progenitor pools, regenerating tapered rays and inherently restoring skeletal geometry primed for future injury responses. 


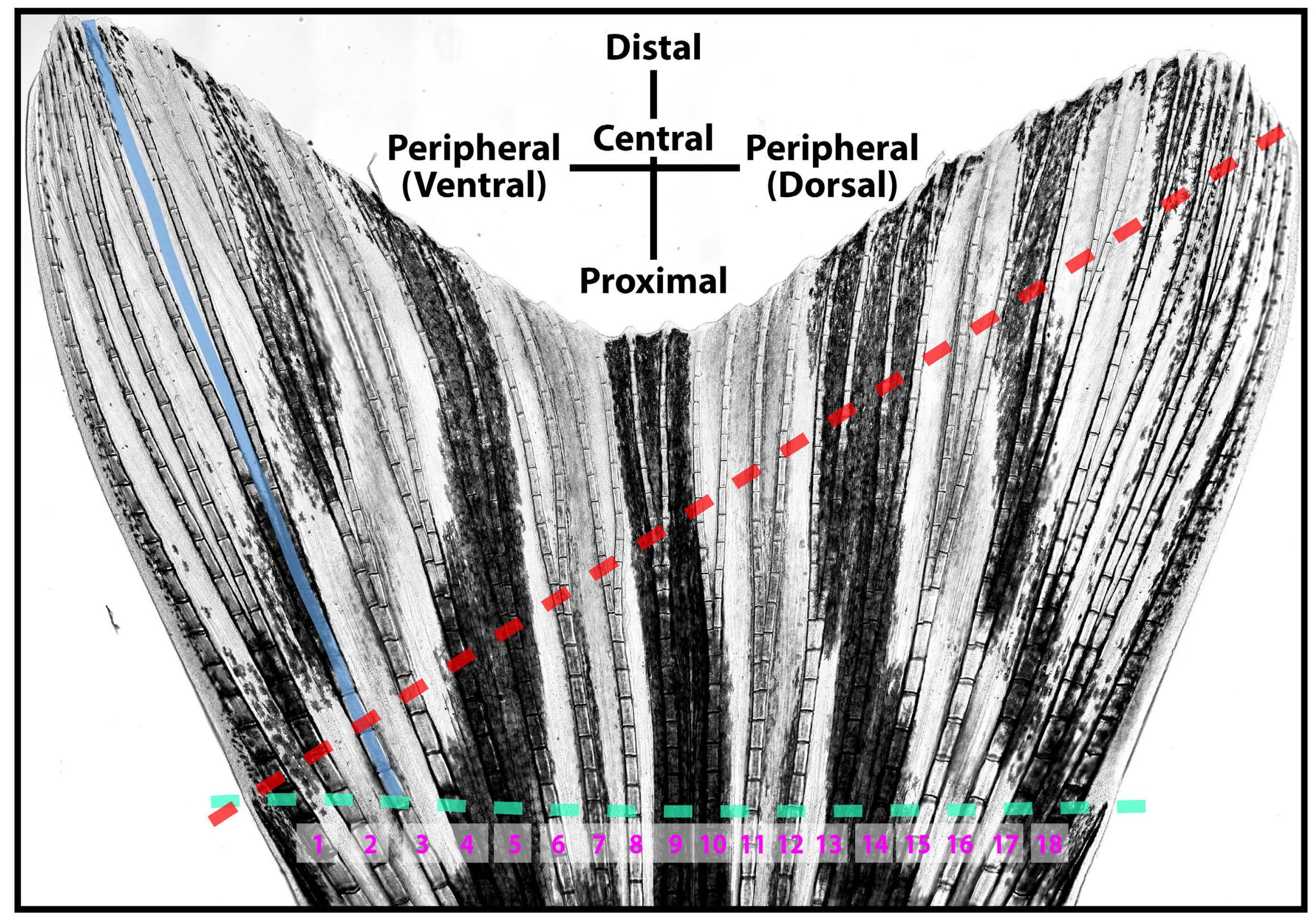

Supplemental Figure 1 


\section{Supplemental Figure 1. A zebrafish caudal fin showing amputation positions and orientation/numbering conventions.}

A high resolution, stitched differential interference contrast (DIC) image of an uninjured adult caudal fin. The dashed green line shows the standard proximal-distal amputation plane spanning

715 between the tips of the un-numbered, truncated peripheral "mini-rays". Diagonal fin amputations follow the dashed red line, starting from the tip of the ventral-most "mini-ray", passing through ray 16's second branch-point, and continuing straight through the dorsal edge. Length measurements (blue line) are from a real or theoretical amputation position to the fin's tip. To accommodate branching, the line is drawn directly in between branches of a mother ray. The

720 terms "central" and "peripheral" distinguish ray position relative to a fin's center or either edge. 
DMSO

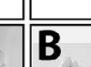

10 nM Wnt-C59

30 nM Wnt-C59

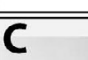
(3)
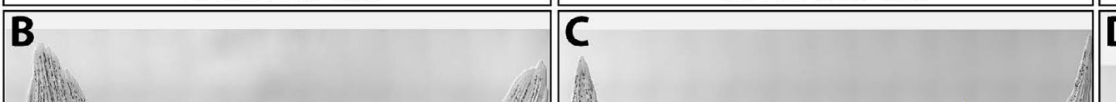

D

100 nM Wnt-C59 || 300 nM Wnt-C59 aire

Supplemental Figure 2 


\section{Supplemental Figure 2. Wnt production correlates with regenerative "demand".}

(A-E) Stitched differential interference contrast (DIC) images of regenerated caudal fins 15 days

725 post amputation (dpa) with exposure to DMSO (A) or indicated concentrations of the Wntsecretion inhibitor Wnt-C59 (B-E). Small molecule exposures began at 4 dpa until imaging at 15 dpa. Yellow and red arrows point to regions with reduced or failed regeneration after drug addition, respectively. The amputation plane is indicated with a dashed red line. Scale bars are 1 $\mathrm{mm}$. 
A

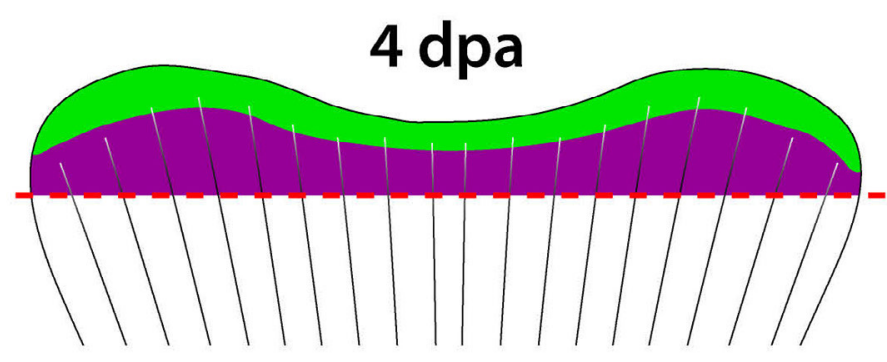

Proximal regenerate

Distal regenerate

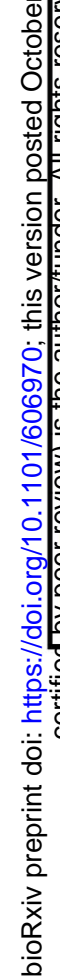

$t b \times 2 b$

foxc1b

mycb

zic2a

dachs

etv $5 b$

zic5

prdmia

etv4

mafa

zic3

tcf7

tcf7 $2 b$

smads

runx

atf3

dach@

sp5a

Supplemental Figure 3
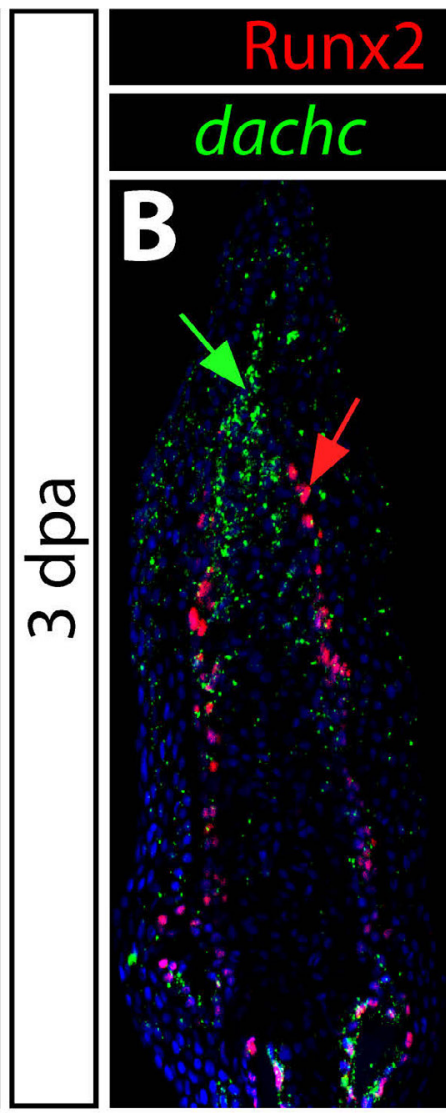

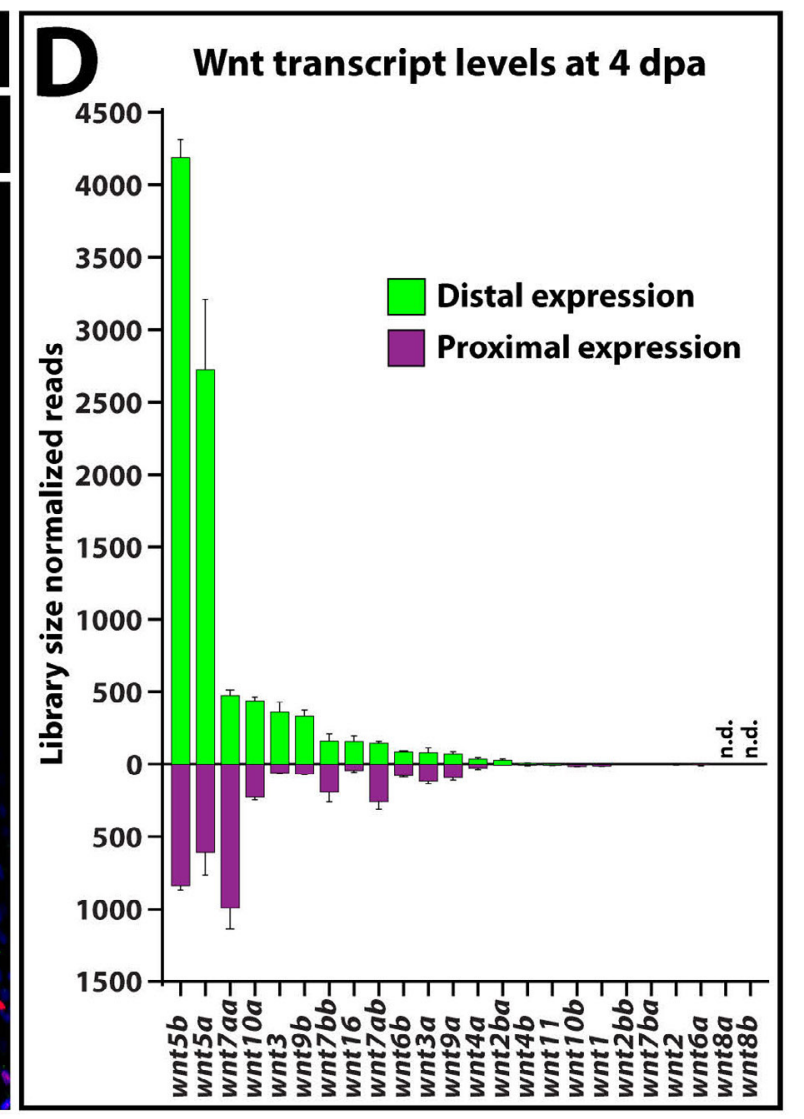




\section{Supplemental Figure 3. dacha and dachc expression defines the distal Wnt-producing} niche.

(A) RNA-Seq analysis comparing distal Wnt-expressing vs. proximal differentiating tissue from 4 day post-amputation (dpa) regenerating fin tissue. The heat map illustrates relative enrichment

735 (red $=$ higher, blue $=$ lower $)$ of differentially expressed transcriptional regulators $(\mathrm{p}<0.05$ and at least 3-fold difference) for each replicate. (B-C) In situ hybridization (in green) for dachc (B) and dacha (C) mRNAs, combined with immunostaining for Runx2 (red) on 3 dpa fin sections. Hoechst-stained nuclei are blue. Red arrows indicate Runx2-expressing osteoblasts that lack dacha/c expression. Green arrows point to the dacha/c-expressing distal niche. (D) Bar graph

740 showing the library size-normalized transcript counts of all 24 zebrafish wnt genes in distal and proximal 4 dpa fin regenerate tissue. The genes are ordered by descending expression levels. $w n t 5 b$ and $w n t 5 a$ have the highest distal transcript levels and are distally enriched. Means of the three replicate pooled samples are shown. Error bars show one standard deviation. 

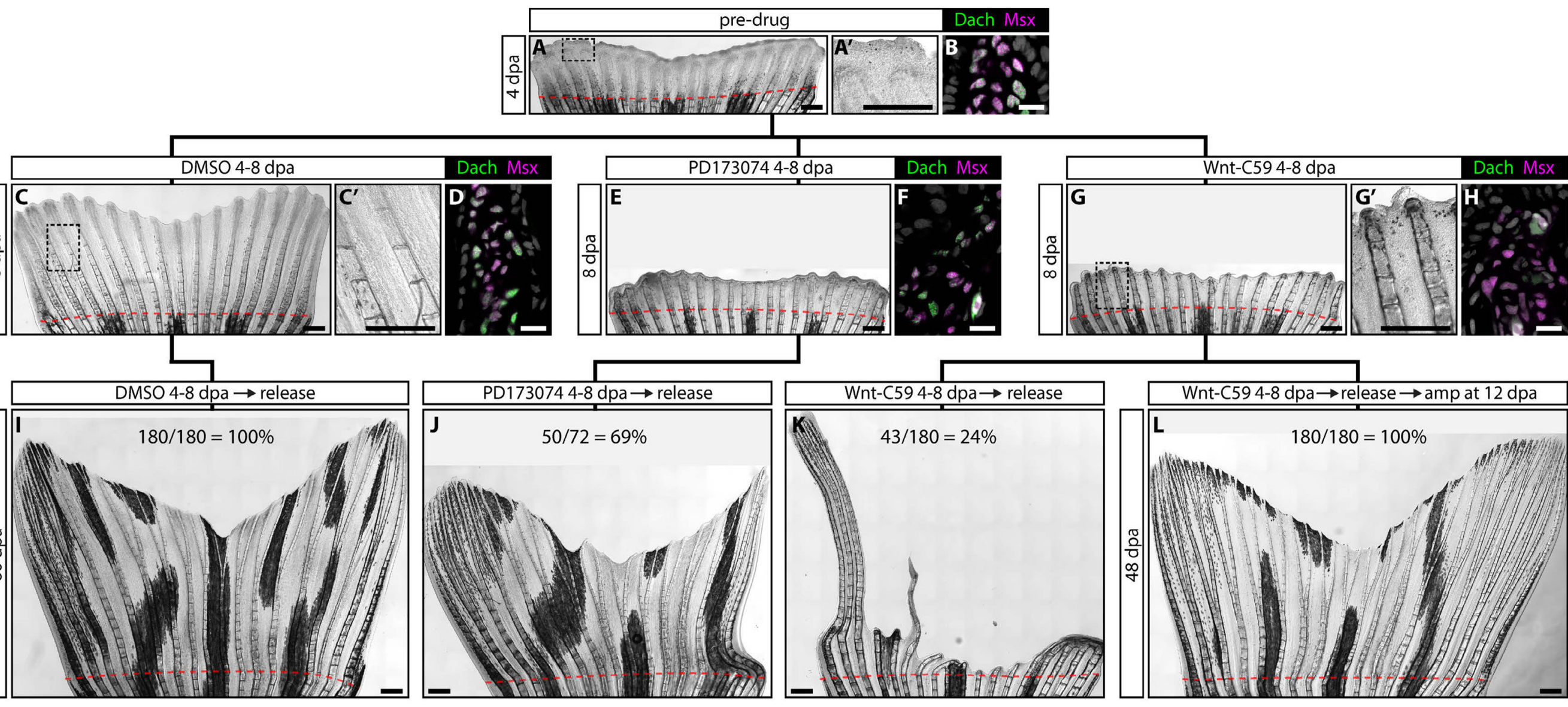

Supplemental Figure 4 


\section{Supplemental Figure 4. Inhibition of Wnt signaling depletes the Dach $^{+}$niche and} irreversibly blocks regeneration.

The black lines connecting figure panels establish a tree diagram showing the experimental workflow. Top row: caudal fins from clutchmate animals are resected and allowed to regenerate through 4 dpa. Middle row: At 4 dpa, animals are treated with DMSO, the FGFR inhibitor

750 PD173074, or the Wnt secretion inhibitor Wnt-C59 until 8 dpa. Bottom row: Animals are then transferred to fish water and allowed to completely regenerate. For (L), fins are re-amputated at 12 dpa proximal to the original amputation site. Whole mount fin images use stitched differential interference contrast (DIC) microscopy. (A) A representative fin at 4 dpa. The region bound by the dashed box is shown at high magnification (A') to emphasize how distal tissue is

755 undifferentiated. (B) A distal regenerate section from a 4 dpa fin immunostained with Dach (green) and Msx (magenta) antibodies. Niche cells express both transcription factors. (C, C', E, G, G') 8 dpa regenerated fins from DMSO (C, C'), PD173074 (E), and Wnt-C59 (G, G') exposed animals. FGF or Wnt inhibition prevents outgrowth past the 4 dpa time of drug addition. However, Wnt is not required for bone differentiation or joint formation (compare G' with A').

760 (D, F, H) 8 dpa sections immunostained with Dach (green) and Msx (magenta) antibodies. Dachexpressing niche cells persist upon FGFR inhibition but are lost when Wnt production is blocked. (I-K) Fin images at 60 dpa after treatment from 4-8 dpa with DMSO (I), PD173074 (J), or Wnt-C59 (K). Unlike FGFR inhibition, Wnt inhibition irreversibly blocks outgrowth. (L) A Wnt-C59 treated animal (from 4-8 dpa) subjected to a secondary amputation at 12 dpa and

765 allowed to regenerate until $48 \mathrm{dpa}$. A secondary amputation effectively "resets" the outgrowth timer by establishing a new niche, allowing regeneration to resume. For (I-L), the number of rays that regenerated normally is shown for each treatment $(n=10$ fish for each condition). A dashed 
bioRxiv preprint doi: https://doi.org/10.1101/606970; this version posted October 2, 2019. The copyright holder for this preprint (which was not certified by peer review) is the author/funder. All rights reserved. No reuse allowed without permission.

red line indicates the amputation plane. Scale bars are $500 \mu \mathrm{m}$ for whole mount images and 25

$\mu \mathrm{m}$ for immunostained sections. 


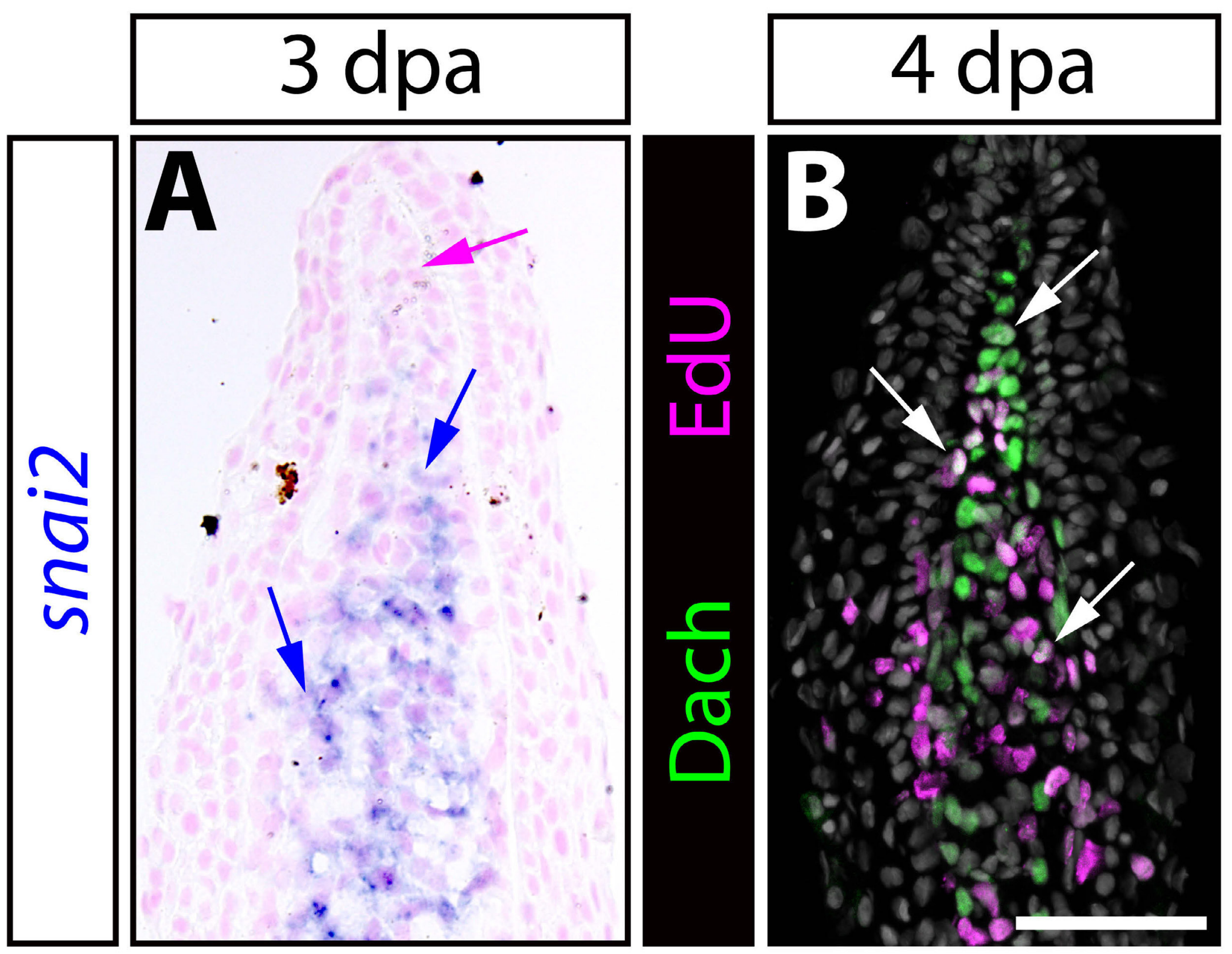

Supplemental Figure 5 


\section{Supplemental Figure 5. Intra-ray mesenchymal cells down-regulate snai2 when transitioning to a Dach ${ }^{+}$niche state that remains proliferative.}

(A) Visualization of snai2 mRNA (in blue) by in situ hybridization on a 3 dpa fin section. The tissue is counterstained with Nuclear Fast Red. Blue arrows indicate snai2-expressing blastema

775 mesenchyme. The magenta arrow highlights the snai2-negative niche. (B) Paraffin section of a 4 dpa regenerating caudal fin stained for EdU incorporation (four hour EdU exposure; magenta) and Dachshund (Dach; green) expression. Nuclei are in grey. Scale bar is $50 \mu \mathrm{m}$. White arrows denote $\mathrm{Edu}^{+} / \mathrm{Dach}^{+}$cells found throughout the niche population. 


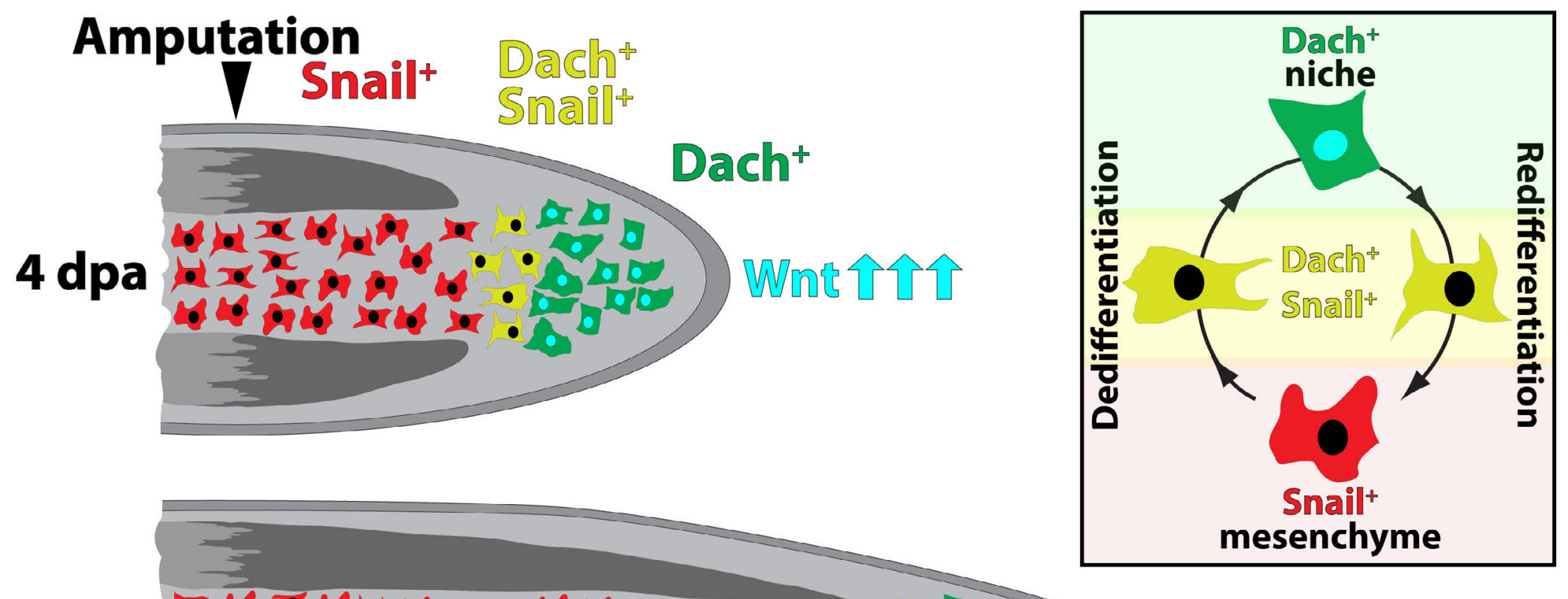

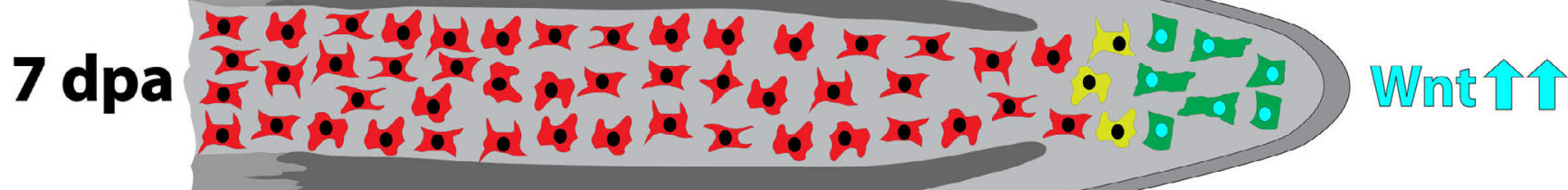

$15 \mathrm{dpa}$ o

Supplemental Figure 6 
780 Supplemental Figure 6. Graphic depicting the progressive depletion of the Wnt-producing, Dach-defined niche population during fin regeneration.

$\operatorname{Dach}^{+}$niche cells originate from Snail-expressing intra-ray mesenchyme released into the forming blastema upon injury. The size of the niche population, and therefore Wnt levels, decreases as the fin regenerates and niche cells transition back into a Snail ${ }^{+}$mesenchymal state. 

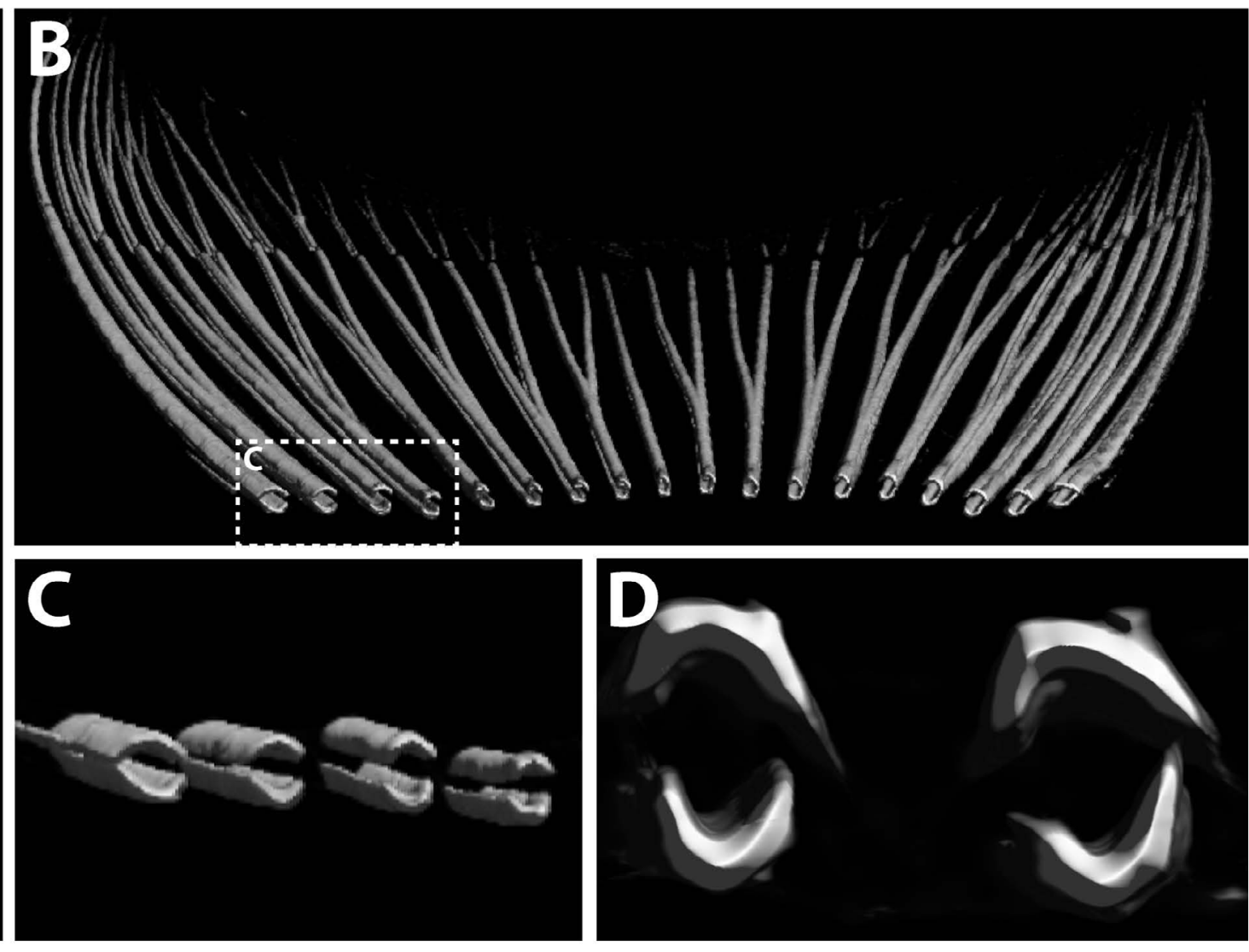

Supplemental Figure 7 


\section{Supplemental Figure 7. Micro-CT analysis shows bony rays across the caudal fin are} variably sized, tapered, and cylindrical. (A-C) 3-D micro-computed tomography images of an adult zebrafish caudal fin highlighting its (A) bony ray skeleton, (B) differentially sized and tapering rays, $(\mathrm{C})$ the cylindrical shape of the intra-ray space between hemi-rays and (D) its

790 approximately circular cross-sectional area. 


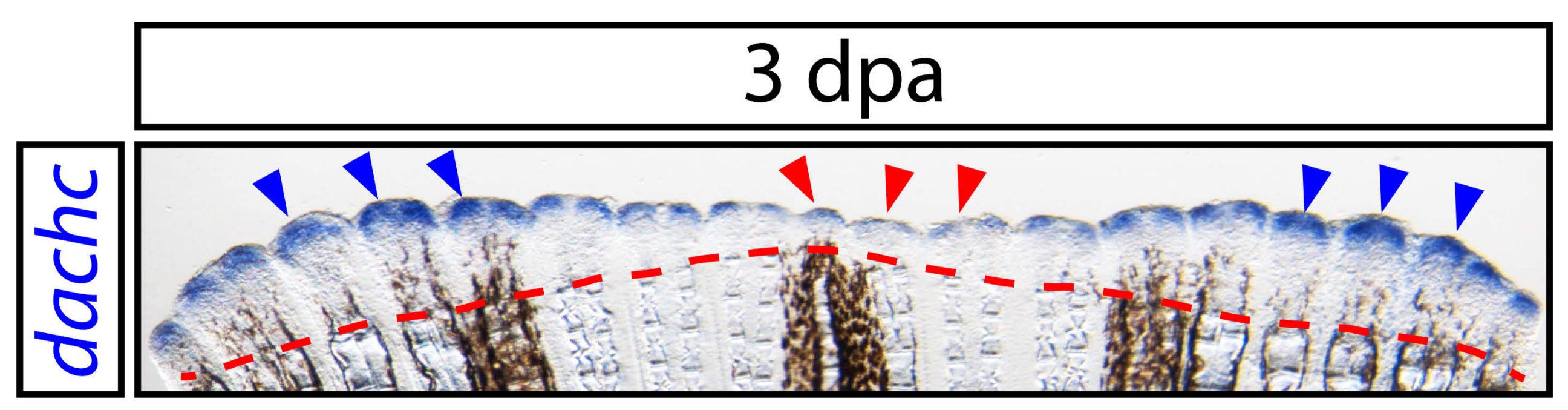

Supplemental Figure 8 
Supplemental Figure 8. Differential niche production across the dorsal-ventral axis during fin regeneration.

Whole mount in situ hybridization on a 3 dpa fin using a dachc probe to visualize distal niche

795 cells (in blue). Blue arrowheads indicate peripheral fin regions with pronounced and broad dachc expression indicative of robust niche cell pools. Red arrowheads point to central fin tissue with modest dachc signal due to relatively small niche cell populations. The dashed red line marks the amputation plane. 
bioRxiv preprint doi: https://doi.org/10.1101/606970; this version posted October 2, 2019. The copyright holder for this preprint (which was not certified by peer review) is the author/funder. All rights reserved. No reuse allowed without permission.

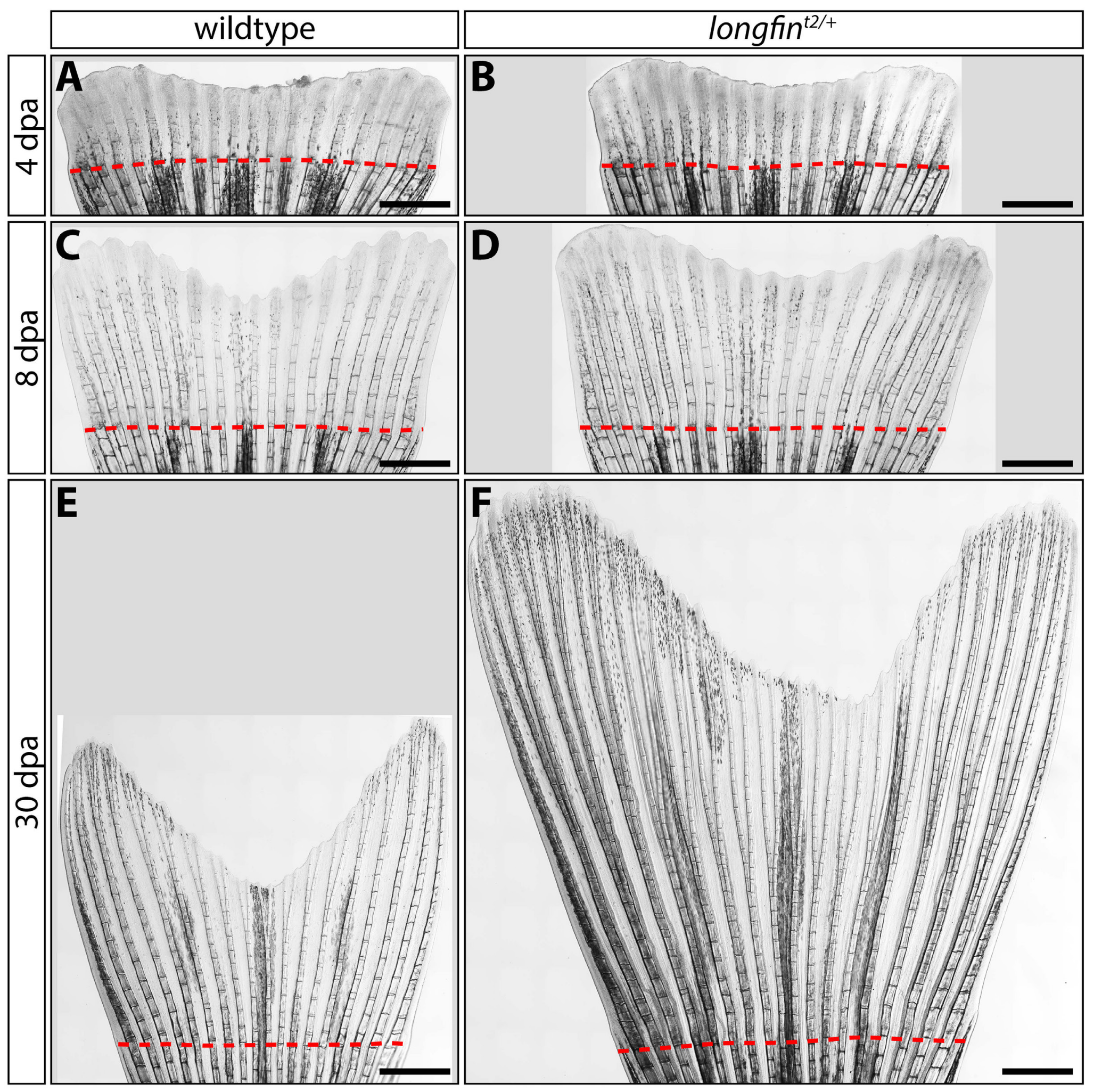


Supplemental Figure 9. Regenerative outgrowth of $\operatorname{longfin}^{t 2 /+}$ caudal fins fails to decelerate. (A-F) Stitched differential interference contrast (DIC) microscope images of caudal fins from clutchmate wildtype and longfin ${ }^{t 2 /+}$ animals at the indicated day post amputation (dpa). Outgrowth is similar through $8 \mathrm{dpa}$ but persists in longfin ${ }^{t 2 /+}$ fish, leading to exceptionally long

805 fins by 30 dpa. Quantitative data for the entire time course $(n \geq 12)$ is shown in Fig. 4. Dashed red lines highlight amputation planes. Scale bars represent $1 \mathrm{~mm}$. 


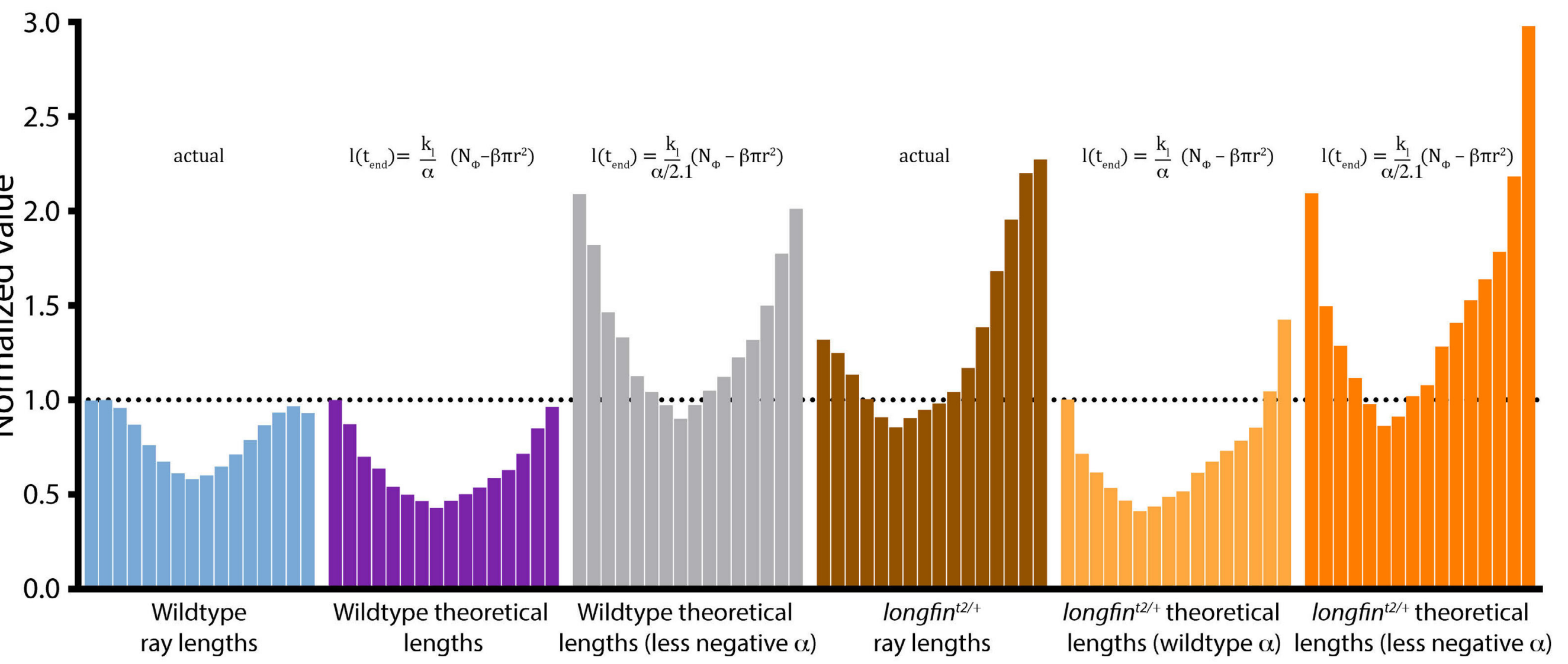

Supplemental Figure 10 


\section{Supplemental Figure 10. longfin fin ray skeletal geometry and modeled regenerative}

810 outgrowth suggests longfin have deficient niche depletion - a broken "countdown timer".

Each distinctly colored set of distributed bars represents actual ray lengths or theoretical regenerated ray lengths for the 16 central rays (from a fixed proximal-distal position). All ray lengths are normalized to the longest wildtype ray. Theoretical lengths are derived from the transpositional scaling equation, which predicts final length as a function of ray radius, with

815 scaling parameters set to restore the widest wildtype ray to its actual length. The alpha parameter $(\alpha)$ then is increased by $2.1 x$ (by dividing $\alpha$, a negative number, by 2.1 ; empirically set by linear regression comparing wildtype and longfin ray area to length relationships) to model a decreased rate of niche depletion that would cause wildtype resected rays to theoretically regenerate to a longfin ${ }^{t 2 /+}$ length. Applying this same increase in $\alpha$ but inputting longfin ${ }^{t 2 /+}$ ray radii into the

820 transpositional scaling formula successfully predicts longfin ${ }^{t 2 /+}$ fin-resected fish would regenerate with a longfin phenotype. 